\title{
Nonequivalent Statistical Equilibrium Ensembles and Refined Stability Theorems for Most Probable Flows
}

\author{
Richard S. Ellis, Kyle Haven, and Bruce Turkington \\ Department of Mathematics and Statistics \\ University of Massachusetts \\ Amherst, MA 01003
}

October 27,2018

\begin{abstract}
Statistical equilibrium models of coherent structures in two-dimensional and barotropic quasi-geostrophic turbulence are formulated using canonical and microcanonical ensembles, and the equivalence or nonequivalence of ensembles is investigated for these models. The main results show that models in which the global invariants are treated microcanonically give richer families of equilibria than models in which they are treated canonically. Such global invariants are those conserved quantities for ideal dynamics which depend on the large scales of the motion; they include the total energy and circulation. For each model a variational principle that characterizes its equilibrium states is derived by invoking large deviations techniques to evaluate the continuum limit of the probabilistic lattice model. An analysis of the two different variational principles resulting from the canonical and microcanonical ensembles reveals that their equilibrium states coincide only when the microcanonical entropy function is concave. These variational principles also furnish Lyapunov functionals from which the nonlinear stability of the mean flows can be deduced. While in the canonical model the well-known Arnold stability theorems are reproduced, in the microcanonical model more refined theorems are obtained which extend known stability criteria when the microcanonical and canonical ensembles are not equivalent. A numerical example pertaining to geostrophic turbulence over topography in a zonal channel is included to illustrate the general results.
\end{abstract}

Keywords: Statistical equilibria; Mean-field theory; Nonlinear stability; Geostrophic turbulence 


\section{Introduction}

A prominent feature of two-dimensional turbulence is the formation of large-scale coherent structures among the small-scale fluctuations of the vorticity field [31, 47]. This selforganization behavior results from the conservation of both energy and enstrophy (the spatial second moment of vorticity) in inviscid, incompressible two-dimensional flow, which causes a net flux of energy toward large scales and a net flux of enstrophy toward small scales [30]. As a consequence, a freely-evolving flow gradually tends toward an equilibrium state consisting of a stable, steady flow on the large scales and disorganized motions on the small scales. This generic behavior is confirmed by numerical simulations of high Reynolds' number flows in various settings. For instance, a freely-decaying flow with doubly-periodic boundary conditions relaxes at long times to either a coherent dipole vortex or double shear layer [39, 48]. Similarly, a weakly driven and dissipated flow is well approximated by a nearly steady coherent structure on the large scales that changes slowly in response to the driving and dissipation [32, 23].

Quasi-geostrophic turbulence behaves in a similar fashion, producing coherent structures on the large scales of motion within a potential vorticity field that is turbulent on a range of small scales [43. In a geophysical context such as the active weather layer on Jupiter, robust mean flows of this kind are observed in the form of persistent jets and spots [34]. Numerous, but less obvious, examples of long-lived mean flows with these general characteristics are also found in the Earth's oceans and atmosphere [41]. Generically, these coherent structures are shear flows or distributed vortices embedded in shear flows.

In this paper we study a statistical equilibrium theory of coherent structures in twodimensional or barotropic quasi-geostrophic turbulence. Several theories of this kind have been proposed and their predictions have been analyzed in some detail; they include the Onsager-Joyce-Montgomery theory of a point vortex gas [40, 28, 29, 8], the Kraichnan energy-enstrophy theory [30, 46, 24, [], and the Miller-Robert theory of a continuum vorticity field [36, 37, 44, 45]. A review and critique of these various theories is given in [49]. In that work it is shown that each of these theories relies upon some explicit or implicit assumptions concerning the form of the random vorticity field on the microscopic scale and that these different assumptions lead to different predictions about the coherent structure on the macroscopic scale. These differences stem from the way in which the generalized enstrophy invariants (the spatial higher moments of vorticity) are included in the various theoretical models. Unlike the global invariants associated with the conservation of energy and circulation, which are "rugged" invariants that depend on the large scales of motion, the generalized enstrophy invariants are "fragile" in the sense that they are sensitive to the vorticity fluctuations on the small scales.

In the present paper we therefore consider a model in which the fragile invariants are replaced by a given probability distribution on the small-scale vorticity fluctuations, which we call the prior distribution. With respect to this underlying probabilistic description of the vorticity field, we then impose the rugged global invariants on the statistical equilibrium 
measure that defines the model. In this fashion we obtain a theory in which a single prior distribution captures the microscopic effects and a few global invariants control the macroscopic features.

Besides being more faithful to the continuum dynamics than the known theories, this model is more easily adapted to realistic physical situations. On the one hand, there can be practical advantages to having a model that utilizes only a few robust invariants, as has been demonstrated in [32, 15]. On the other hand, a suitable prior distribution can be fit directly to the one-point vorticity statistics available from numerical simulations or physical data. Alternatively, it can be inferred indirectly by comparing the predicted vorticity-streamfunction profile with an observed profile.

In the context of a model of this kind, we have the choice of building the equilibrium statistical measure from a canonical ensemble or from a microcanonical ensemble with respect to the rugged invariants. In most applications of statistical mechanics these alternative formalisms define equivalent theories that have identical equilibrium states in the thermodynamic limit [2, 3]. It is rather surprising, therefore, to discover that in our models of coherent structures the two ensembles are not always equivalent. In fact, we find that there are regimes in which the equilibrium states for the microcanonical ensemble are entirely omitted by the canonical ensemble. Moreover, numerical computations based on the microcanoncial model show that these regimes often contain mean flows of great physical interest. In essence, the reason for this novel behavior lies in the character of the statistical equilibrium models: they are local mean-field theories in which the continuum limit is nonextensive, the interactions are long-range, and the inverse temperature is negative.

Given that some equilibrium states for microcanonical model are not realized by the corresponding canonical model, we are led to ask whether these most probable states correspond to stable flows. We answer this question in the affirmative by proving that all nondegenerate canonical and microcanonical equilibrium states define nonlinearly stable, steady mean flows. In the canonical model, these results reduce to the well-known the Arnold stability theorems, which rely on Lyapunov functionals constructed from the rugged invariants and the information (negative entropy) functional associated with the prior distribution [1], 33]. In the microcanonical model, however, these standard Lyapunov functionals are not positive definite at those equilibrium states which are not realized by the canonical model. In the nonequivalent case we instead use a new class of Lyapunov functionals to demonstrate the stability of the most probable flows for the microcanonical model. In this construction we introduce a penalization of the standard functional with respect to the microcanonical constraints that makes the resulting Lyapunov functional positive definite at the microcanonical equilibrium states. Such penalized functionals are identical with the so-called augmented Lagrangians used in methods for constrained optimization [4], 38].

These results support our contention that the natural formulation of a statistical equilibrium model of coherent structures is the one in which conservation of generalized enstrophy is relegated to a prior distribution, and conservation of energy and circulation are imposed microcanonically. From a mathematical standpoint, this model is preferrable to the cor- 
responding canonical model because, in general, its family of equilibrium states is richer. From a physical point of view, the microcanonical conditions are pertinent because the energy and circulation are trapped in the largest scales of motion, and hence these rugged invariants are isolated from interactions with larger systems or ignored degrees of freedom. Reciprocally, the use of a prior distribution on the vorticity, which amounts to a canonical treatment of the generalized enstrophy invariants, acknowledges that the statistical properties of the vorticity on the small scales are determined by contact with a bath of unresolved turbulent motions. Finally, our refined stability theorems ensure that the most probable flows defined by the model are nonlinearly stable for any admissible values of the microcanonical constraints, even when the classical sufficient conditions for stability are not satisfied.

The paper is organized as follows. In Section 2 we formulate a general equilibrium statistical model that includes two-dimensional and barotropic geostrophic turbulence with topography. After explaining the role of the prior distribution in the probabilistic lattice model, we construct the canonical and microcanonical models, respectively. In Section 3 we then present the variational principles for these two models in the continuum limit as the lattice spacing tends to zero. Our analysis makes use of large deviation techniques, which are uniquely suited to derivations of this kind [18, 19]. In particular, we introduce a coarsegraining of the microscopic vorticity field and present the fundamental large deviation principle that this process satisfies. In another paper we state and prove a general theorem that contains this result as a special case 21]. On the basis of this result, we develop the variational principles governing the equilibrium macrostates in the canonical and the microcanonical continuum models. We give the complete proofs of the large deviation estimates needed to justify these variational principles in a companion paper [20], where we treat an general class of models defined in terms of local mean-field interactions. In Section 4 we turn to the equivalence of ensembles questions, invoking ideas from convex analysis and constrained optimization theory to obtain sharp and complete results. A more general treatment of these issues is also presented in the companion paper [20]. In Section 5 we present the nonlinear stability theorems, first reviewing the known theorems that pertain to the canonical model and then developing the refinement of those theorems that applies to the microcanonical model. Finally, in Section 6 we display the results of some numerical solutions to the microcanonical variational principle for barotropic shear flows over a zonal topography. In this physically interesting problem the nonequivalenceof-ensembles behavior is quite conspicuous.

Our presentation throughout this paper is a synthesis of physical modeling and mathematical analysis, which is intended to focus on the conceptual aspects of the models we study. With this goal in mind, we omit many of the technical details and proofs, referring the reader to our other papers [5, 20, 21] for those aspects. 


\section{Formulation of the models}

2.1 Two-dimensional and geostrophic turbulence. For the microscopic dynamics that underlies our statistical equilibrium models we adopt an equation of motion that contains as special cases the governing equations for both purely two-dimensional turbulence and barotropic quasi-geostrophic turbulence. Namely, we consider the nonlinear advection equation

$$
\frac{\partial Q}{\partial t}+\frac{\partial Q}{\partial x_{1}} \frac{\partial \psi}{\partial x_{2}}-\frac{\partial Q}{\partial x_{2}} \frac{\partial \psi}{\partial x_{1}}=0
$$

in which $Q=Q\left(x_{1}, x_{2}, t\right)$ and $\psi=\psi\left(x_{1}, x_{2}, t\right)$ are real scalar fields related by the elliptic equation

$$
Q=-\Delta \psi+r^{-2} \psi+b .
$$

In this defining equation, $\Delta=\partial^{2} / \partial x_{1}^{2}+\partial^{2} / \partial x_{2}^{2}$ denotes the Laplacian on $R^{2} ; r$ is a given positive constant which may be infinity; and $b=b\left(x_{1}, x_{2}\right)$ is a specified continuous function. The flow velocity field $v$ is nondivergent and is determined from the streamfunction $\psi$ by $v=\left(\partial \psi / \partial x_{2},-\partial \psi / \partial x_{1}\right)$. For the sake of definiteness, we take the flow domain to be a channel

$$
\mathcal{X}=\left\{x=\left(x_{1}, x_{2}\right):\left|x_{1}\right|<\ell_{1} / 2,\left|x_{2}\right|<\ell_{2} / 2\right\}
$$

with a period length $\ell_{1}$ and finite width $\ell_{2}$. The boundary conditions for ideal flow in such a channel are achieved by setting $\psi=0$ on the walls $x_{2}= \pm \ell_{2} / 2$ and by imposing $\ell_{1}$-periodicity in $x_{1}$.

Equations (11)-(2) reduce to the Euler equations governing incompressible, inviscid flows in two dimensions when $r=\infty$ and $b=0$. In this case $Q$ coincides with the vorticity $\omega=\partial v_{2} / \partial x_{1}-\partial v_{1} / \partial x_{2}$. In such a flow the conservation of momentum is equivalent to the exact rearangement of vorticity $\omega$ under the area-preserving flow maps for the velocity field $v$ induced instantaneously by $\omega$.

When a finite $r$ and a nonvanishing $b$ are included in (11)-(2), these general equations contain the standard equations governing a shallow rotating layer of homogeneous incompressible inviscid fluid in the limit of small Rossby number. In the geophysical literature where these equations are derived and discussed [41], the nondimensionalized spatial variables $\left(x_{1}, x_{2}\right)$ are written as $(x, y)$ and the geostrophic streamfunction $\psi$ is replaced by $-\psi$, which also represents the nondimensionalized free-surface perturbation. Under appropriate quasi-geostrophic scalings and up to first-order in the Rossby number, the flow is nondivergent and its potential vorticity $Q$, defined by (2), is advected by the flow according to (1). The inhomogeneous term in (2) is given by $b=\beta y+h$, where $\beta$ is the gradient of the Coriolis paramter $f=f(y)$ and $h$ is the height of the bottom topography. The constant $r$ in (2) is the Rossby deformation radius $r=\sqrt{g H_{0}} / f_{0}$, which is determined by the gravitational acceleration $g$, the mean fluid depth $H_{0}$, and a mean value $f_{0}$. We refer the reader to the literature for a complete discussion of these fundamental equations and their properties 41. 
The general equations (11)-(2) also contain the governing equations for the so-called 1-1/2 layer model, in which a shallow upper layer lies on a deep lower layer of denser fluid whose motion is unaffected by that in the upper layer. Besides having oceanographic applications, this model is often used to describe the observed weather layer of the Jovian atmosphere [26, 34]. In the applications to Jupiter, the lower layer flow is assumed to be steady, zonal and geostrophically balanced. Then the potential vorticity for the active upper layer is given by (2) with $b=\beta y-r^{-2} \psi_{2}(y)$, where $\psi_{2}$ denotes the streamfunction for the flow in the lower layer. In this way, the deep flow produces an effective bottom topography. The appropriate Rossby scale $r$ is determined as in the single layer model, except that a reduced gravity $g^{\prime}$ is used. With these choices, (1)-(2) govern the quasi-geostrophic dynamics of the shallow upper layer.

¿From the point of view of statistical equilibrium theory, the underlying continuum dynamics dictated by (11)-(2) serves as a mechanism for mixing the scalar field $Q$ subject to the constraints imposed by the various conserved quantities for that dynamics. Indeed, the equilibrium statistical models that we study are constructed by postulating that the underlying dynamics is ergodic with respect to the ideal invariants. This ergodic hypothesis is not expected to be universally valid. Nevertheless, numerous observations and simulations of two-dimensional and geostrophic turbulence show that typically the self-induced straining of the advected field $Q$ leads to an effective randomization of $Q$. For instance, in a free evolution from a generic smooth field $Q^{0}, Q$ develops local finite-amplitude fluctuations on a range of small scales as time progresses. This behavior is related to the direct cascade of enstrophy to small scales. At the same time, $Q$ tends to organize into coherent vortices at the large scales, and these vortices gradually merge into a final steady state. This dual behavior is associated with the inverse cascade of energy to large scales. The goal of the statistical equilibrium models is to characterize the typical steady mean flows that persist on the large scales without resolving the small scales of motion. The validity of these models must be checked a posteriori from their predictions, since a priori tests or proofs of the ergodic hypothesis are generally not feasible.

The conserved quantities associated with (1)-(2) are the total energy $H$, the total circulation $C$, and a family of generalized enstrophies $A$, given by,

$$
\begin{gathered}
H=\frac{1}{2} \int_{\mathcal{X}}\left[\left(\frac{\partial \psi}{\partial x_{1}}\right)^{2}+\left(\frac{\partial \psi}{\partial x_{2}}\right)^{2}+r^{-2} \psi^{2}\right] d x \\
C=\int_{\mathcal{X}}[Q-b] d x \\
A=\int_{\mathcal{X}} a(Q) d x
\end{gathered}
$$

where $a$ is an arbitrary, sufficiently smooth, real function on the range of $Q$. In addition, the $x_{1}$-component of linear impulse (momentum) $M$ is also conserved in the channel geometry 
that we consider; it is given by

$$
M=\int_{\mathcal{X}} x_{2}[Q-b] d x
$$

We note that the expression $Q-b=\zeta+r^{-2} \psi$ appearing in the circulation and impulse integrals is a sum of the relative vorticity, $\zeta=-\Delta \psi$, and the vortex stretching term, $r^{-2} \psi$, due to deformation of the free-surface.

While each of these quantities is precisely conserved by the continuum dynamics, the role that $H, C$ and $M$ play in the statistical equilibrium models differs dramatically from that played by the nonlinear enstrophies $A$. This crucial difference is a consequence of the fact that the generalized enstrophy invariants are sensitive to the small-scale structure of $Q$, while the energy, circulation, and impulse invariants depend on the large scales of motion. For this reason, in the following two subsections we formulate the various models by first defining the probabilistic structure of the small scales and then introducing the conditioning determined by the global invariants for the large scales.

2.2 Generalized enstrophies and small-scale fluctuations. In order to define our continuum models, we first replace the infinite dimensional phase space of continuum vorticity fields $Q$ by a sequence of finite dimensional phase spaces and then take an appropriate continuum limit. To this end, we introduce a lattice $\mathcal{L}$ on the domain $\mathcal{X}$ having $n$ sites and construct a probabilistic lattice model for each $n$. It suffices to use a uniform intersite spacing in both the $x_{1}$ and $x_{2}$ directions; say, a dyadic partition of the intervals $-\ell_{1} / 2<x_{1}<\ell_{1} / 2$ and $-\ell_{2} / 2<x_{2}<\ell_{2} / 2$ into $2^{m_{1}}$ and $2^{m_{2}}$ equal parts, so that $n=2^{m_{1}+m_{2}}$. The domain $\mathcal{X}$ then consists of the disjoint union of $n$ microcells $M(s)$ indexed by the sites $s$ in the lattice $\mathcal{L}$. The phase space for the lattice model is the product space $\Omega_{n}=R^{n}$, the microstates in the lattice model being points in $\Omega_{n}$. We identify these micrstates with vorticity fields $Q$ that are piecewise-constant relative to $\mathcal{L}$; that is, $Q(x)=Q(s)$ for all $x \in M(s), s \in \mathcal{L}$. For the sake of simplicity, we shall use the same notation for the continuum field $Q(x), x \in \mathcal{X}$, governed by the underlying partial differential equations and the discretized field $Q(s), s \in \mathcal{L}$, in the lattice model.

The small-scale fluctuations of the microstates in the lattice model are described by the product measure

$$
\Pi_{n}(d Q)=\prod_{s \in \mathcal{L}} \rho(d Q(s)) \quad \text { on } \quad \Omega_{n}
$$

in which $\rho(d y)$ is a given probability distribution on $R$. Here and throughout the paper, $y$ denotes a real variable running over the range of $Q$. With respect to the probability distribution $\Pi_{n}$ the microscopic fields $Q$ consist of $n$ independent, identically distributed random variables over the $n$ microcells in the lattice. We refer to the common distribution $\rho$ as the prior distribution, signifying that it describes the statistical properties of the microstate $Q$ before the conditioning due to the rugged invariants is imposed. 
When $\rho(d y)=e^{-a(y)} d y, y \in R$, for some continuous function $a$ on $R$, the product measure $\Pi_{n}$ in (8) coincides with canonical Gibbs measure with respect to $A \doteq \frac{1}{n} \sum a(Q(s))$, which is the discetization of the generalized enstrophy integral $A=\int a(Q) d x$. That is,

$$
\Pi_{n}(d Q)=e^{-n A_{n}(Q)} \prod_{s \in \mathcal{L}} d Q(s)=\prod_{s \in \mathcal{L}} e^{-a(Q(s))} d Q(s) .
$$

In light of this identity, the role of $\Pi_{n}(d Q)$ in the lattice model is evident from the general principles of statistical mechanics: it is the most probable distribution on $\Omega_{n}$ with respect to the phase volume $d Q=\prod d Q(s)$ that is consistent with the conservation of generalized enstrophy $A_{n}$. Typically, this characterization of the canonical ensemble is justified by two dynamical properties: 1) the invariance under the phase flow of the phase volume $d Q ; 2$ ) the dynamical invariance of the function $A_{n}$. In the models we study, however, a lattice dynamics discretizing the underlying continuum dynamics for which these two properties hold is not known. Consequently, it is necessary to treat the construction of the product measure $\Pi_{n}(d Q)$ as a modeling issue, justifying its choice on whatever theoretical results are available and whatever practical considerations are at hand.

The principal reason for preferring the canonical ensemble $\Pi_{n}(d Q)$ to the corresponding microcanonical ensemble is the sensitivity of the generalized enstrophies $A$ to small-scale motions. In physical terms $\Pi_{n}(d Q)$ describes a random field $Q$ on the lattice $\mathcal{L}$ in which there is a coupling between the fluid motions on scales resolved by the lattice and the unresolved turbulence on smaller scales. As in standard statistical equilibrium theory, the canonical formulation is appropriate to a system coupled to a reservoir, or thermal bath [2, 3]. The prior distribution $\rho$ that parametrizes $\Pi_{n}(d Q)$ is effectively a generalized inverse temperature for the potential vorticity fluctuations on the lattice microscale. By contrast, the microcanonical ensemble based on a (finite or infinite) family of generalized enstrophies $A_{n}$ enforces the exact conservation of each $A_{n}$ on the lattice, inhibiting the exchange of generalized enstrophy between the resolved scales and the unresolved scales. The well-known flux of enstrophy to small scales therefore invalidates the microcanonical formulation.

Statistical equilibrium theories of the long-time average behavior of solutions to (11)(2) have tended to emphasize the microcanonical formulation. Originally, Miller [36, 37] and Robert 44, 45 independently constructed a model by assuming that the exact rearrangement of vorticity under the continuum dynamics is imitated on the lattice $\mathcal{L}$ by an unspecified lattice dynamics. Under this assumption all generalized enstrophies $A_{n}$ are exactly conserved in the lattice model. This approach produces a well-defined model in which the complete family of vorticity invariants is imposed microcanonically. Later, Turkington [49, 5] criticized the assumption made in the Miller-Robert model and formulated a modification of it that is derived instead from the underlying exact continuum dynamics on $\mathcal{X}$. In the Turkington model, the evolution of the continuum vorticity field is observed on the lattice $\mathcal{L}$ by averaging over the scales smaller than the lattice microscale, and consequently the family of equality constraints on all generalized enstrophies imposed in the 
Miller-Robert model is replaced by a weaker family of inequality constraints on all convex enstrophies. This approach results in a model that accounts for the partial loss of the nonlinear enstrophies to submicroscale fluctuations. Among statistical equilibrium models that associate a final coherent state with a given initial state this model is the most faithful to the underlying ideal continuum dynamics.

For the reasons mentioned above, however, a canonical formulation with respect to the generalized enstrophies usually furnishes a more appropriate physical model than a microcanonical formulation. Moreover, the equilibrium equations for the Turkington model are isomorphic to those for the canonical model with a prior distribution (8) under the identification $\rho(d y)=e^{-a(y)} d y$; in the microcanonical case the function $a$ is determined by the Kuhn-Tucker multipliers for the family of convex enstrophy inequalities, while in the canonical case it is prescribed 49, 5]. Whether all microcanonical equilibria are realized as canonical equilibria is not known.

In practical applications these statistical equilibrium models are used to produce families of most probable large-scale flows that coexist with other complex mechanisms influencing the small-scale motions. Under these circumstances the canonical ensemble (8) is often desirable because the prior distribution $\rho$ can be used to model the one-point probability distribution of the vorticity fluctuations. On the other hand, the constraints on generalized enstrophies, or potential vorticity moments, are of dubious relevance in these realistic situations. For instance, in two-dimensional turbulence with weak driving and small dissipation it is possible to invoke a statistical equilibrium model as an adiabatic approximation to the evolution of the large-scale structure [32, 23, 14]. In these applications only the lowest-order moments of vorticity are sufficiently robust to be retained in the model. Similarly, comparisons with direct numerical simulations of freely-decaying turbulence show good agreement with the predictions of the model only when the higher-order moments of vorticity is altered to account for dissipation [6]. These tests show that it is necessary to take a prior distribution that is compatible with the relaxed final state. In the context of geostrophic turbulence, the modeling of the turbulent small scales is further complicated by the possible effects of nonvanishing Rossby and Froude numbers 42. Given the asymptotic nature of the quasi-geostrophic equations themselves, it is reasonable to fit the prior distribution to available data. In Section 6, we briefly indicate how this empirical approach can be used to formulate a model of zonal jets in a Jovian atmosphere.

For the purposes of our general discussion throughout Sections 3, 4 and 5, we let the prior distribution $\rho$ be an arbitrary probability distribution on $R$ subject only to the decay condition (11), and we base all of our models on the canonical ensemble (8) parametrized by such $\rho$. This simple choice of the product measure $\Pi_{n}(d Q)$ is natural in the context of statistical equilibrium theory. Any better choice would require a new theory of the correlation structure of turbulent scales, derived presumably from nonequilibrium considerations.

2.3 Global invariants and large-scale motions. The statistical equilibrium lattice models that we consider are constructed by imposing the global invariants $H$ and $C$ on the 
product measure $\Pi_{n}(d Q)$. In this construction we can consider either the canonical or the microcanonical ensemble with respect to these invariants. A main goal of this paper is to investigate the equivalence or nonequivalence of these two different ensembles. Accordingly, we now proceed to formulate these canonical and microcanonical models.

The canonical model is defined by the Gibbs distribution

$$
P_{n, \beta, \gamma}(d Q)=Z_{n}(\beta, \gamma)^{-1} \exp \left(-n \beta H_{n}(Q)-n \gamma C_{n}(Q)\right) \Pi_{n}(d Q),
$$

and is parametrized by $\beta, \gamma \in R$, which play the roles of "inverse temperature" and "chemical potential," respectively. The partition function

$$
Z_{n}(\beta, \gamma)=\int_{\Omega_{n}} \exp \left(-n \beta H_{n}(Q)-n \gamma C_{n}(Q)\right) \Pi_{n}(d Q)
$$

normalizes the probability distribution $P_{n, \beta, \gamma}(d Q)$ on $\Omega_{n}$. We use the traditional notation $\beta$ for inverse temperature even though this symbol overlaps with that used in the geophysical literature for the gradient of the Coriolis parameter; we expect that the distinction will be clear enough from context.

The microcanonical model is defined by the conditional distribution

$$
P_{n}^{E, \Gamma}(d Q)=\Pi_{n}\left\{d Q \mid H_{n}(Q)=E, C_{n}(Q)=\Gamma\right\},
$$

at given values $E$ and $\Gamma$ of the global invariants. For technical reasons, it is necessary to replace the exact equality $H_{n}=E$ in (10) by a containment $H_{n} \in[E-\epsilon, E+\epsilon]$ for a small finite $\epsilon>0$ and similarly for the exact equality $C_{n}=\Gamma$. For the sake of clarity of exposition, however, we will ignore this technical point and set $\epsilon=0$ throughout our discussion, leaving the obvious adjustments to the reader.

The functionals $H_{n}$ and $C_{n}$ in (9) and (10) are the lattice versions of the functionals $H$ and $C$ defined on the continuum field $Q$ in (4) and (5), respectively. $H_{n}$ and $C_{n}$ act on $\Omega_{n}$ by identifying each microstate $Q \in \Omega_{n}$ with the corresponding piecewise-constant function $Q \in L^{2}(\mathcal{X})$, and by evaluating the functionals $H$ and $C$ on that field; the corresponding solution $\psi$ to (2) then determines $H(Q)$. Some straightforward calculations show that they have the explicit expressions

$$
\begin{gathered}
H_{n}(Q)=\frac{\ell_{1}^{2} \ell_{2}^{2}}{2 n^{2}} \sum_{s \in \mathcal{L}} \sum_{s^{\prime} \in \mathcal{L}} g_{n}\left(s, s^{\prime}\right) Q(s) Q\left(s^{\prime}\right)-\frac{\ell_{1} \ell_{2}}{2 n} \sum_{s \in \mathcal{L}} h_{n}(s) Q(s), \\
C_{n}(Q)=\frac{\ell_{1} \ell_{2}}{n} \sum_{s \in \mathcal{L}} Q(s)-b(s),
\end{gathered}
$$

where $g_{n}\left(s, s^{\prime}\right)$ is the average over $M(s) \times M\left(s^{\prime}\right)$ of the Green function $g\left(x, x^{\prime}\right)$ defined by $\left(-\Delta+r^{-2}\right) g=\delta\left(x-x^{\prime}\right)$, and $h_{n}(s)$ is the average over $M(s)$ of the solution $h(x)$ to $\left(-\Delta+r^{-2}\right) h=b(x)$; both $g\left(x, x^{\prime}\right)$ and $h(x)$ satisfy the boundary conditions on $\partial \mathcal{X}$ imposed 
on $\psi(x)$. The lattice energy $H_{n}$ consists of a quadratic self-interaction term with a potential $g_{n}$ and a linear term involving $h_{n}$ that represents interaction with the bottom topography.

It is important to note that the vortex self-interactions governed by $H_{n}$ are long-range, being determined essentially by the Green function $g\left(x, x^{\prime}\right)$ for the partial differential operator $-\Delta+r^{-2}$ on $\mathcal{X}$. This property, combined with the form of the product prior distribution $\Pi_{n}(d Q)$, gives these statistical equilibrium models their character as local mean-field theories. Moreover, the long-range interactions imply that the energy function $H_{n}$ is a rugged invariant, meaning that it is not sensitive to the small-scale structure of the vorticity field. Indeed, $H_{n}$ depends only on the local average of $Q$ in a neighborhood of any point, and therefore it is well approximated a spatial coarse-graining of $Q$. The same properties are shared by $C_{n}$ because it is a linear function of $Q$. By contrast, as stressed in the preceding subsection, all nonlinear enstrophies $A_{n}$ are fragile invariants in the sense that they cannot be approximated by their values on a coarse-grained state.

The canonical parameters $\beta$ and $\gamma$ are scaled by a factor $n$ in (9). This scaling ensures that, in the continuum limit as $n \rightarrow \infty$, the mean values $\left\langle H_{n}\right\rangle$ and $\left\langle C_{n}\right\rangle$ with respect to this canonical ensemble tend to finite limits, and that the variances of $H_{n}$ and $C_{n}$ around these mean values tend to zero. The canonical ensemble (9) thus produces equilibrium states having finite total energy and total circulation in the continuum limit, and hence it is compatible with the microcanonical ensemble (10) in which $E$ and $\Gamma$ are fixed and finite as $n \rightarrow \infty$. We note that, while this scaling of the parameters determining the canonical ensemble is natural in these local mean-field models, it results in a nonextensive continuum limit that is different from the usual thermodynamic limit [37].

The linear impulse invariant $M$, which is associated with the translational symmetry of the channel domain, can also be included in either the canonical or the microcanonical ensembles. For the sake of clarity, however, we ignore it in our development. If $M$ is treated canonically, then the energy function $H_{n}$ is simply replaced by $H_{n}+U M_{n}$, where $(U, 0)$ is the velocity of a given uniform zonal flow. Alternatively, if $M$ is imposed microcanonically, then $U$ is determined implicitly. In either formulation, the analysis of the impulse constraint is the same as that of the circulation constraint, which is also linear in $Q$.

\section{Maximum entropy principles}

In this section we investigate the continuum limit of the canonical and microcanonical models constructed in the preceding section, and we thereby derive the maximum entropy principles which characterize the most probable states for those models. Our analysis of the continuum limit relies on the powerful methods of the theory of large deviations 18, 12. First, we establish a large deviation principle for a certain coarse-graining of the potential vorticity field $Q$ with respect to the product prior distribution $\Pi_{n}(d Q)$. With this basic result in hand, we then analyze the canonical ensemble $P_{n, \beta, \gamma}$ and the microcanonical ensemble $P_{n}^{E, \Gamma}$, and establish large deviation principles for the coarse-grained field with 
respect to each of these ensembles. In this way we obtain a variational characterization of the equilibrium macrostates for each model.

3.1 Coarse-grained process. We now introduce a macroscopic description of the potential vorticity field that complements the microscopic description inherent in the lattice model. We take the space of macrostates $q$ to be the Hilbert space $L^{2}(\mathcal{X})$ with the usual norm $\|q\|^{2}=\int_{\mathcal{X}} q^{2} d x$. This natural and convenient choice requires us to impose a certain decay condition on the prior distribution $\rho$. Specifically, we assume that there exists $\delta>0$ such that

$$
\int_{R} \exp \left(\frac{\delta}{2}|y|^{2}\right) \rho(d y)<\infty .
$$

Since this decay condition holds for most prior distributions of interest, including compactly supported and Gaussian distributions, we adopt it for the sake of simplicity throughout Sections 3, 4 and 5. In Section 6, however, we relax it for a particular prior distribution used in the numerical example.

In order to establish the connection between the microscopic and macroscopic levels of description, we define a certain coarse-grained process as follows. Partition the domain $\mathcal{X}$ into $\tilde{n}=2^{r_{1}+r_{2}}$ macrocells $\mathcal{X}_{j_{1}, j_{2}}$, with $r_{1} \ll m_{1}, r_{2} \ll m_{2}$, and $j_{1}=1, \ldots, 2^{r_{1}}, j_{2}=$ $1, \ldots, 2^{r_{2}}$. This partition represents a coarsening of the lattice $\mathcal{L}$ that defines the phase space $\Omega_{n}$; each of the $\tilde{n}$ macrocells $\mathcal{X}_{j_{1}, j_{2}}$ contains $n / \tilde{n}$ sites of $\mathcal{L}$. Now, let $\tilde{Q}_{n, \tilde{n}}$ be the $L^{2}(\mathcal{X})$-valued stochastic process defined by averaging the random microstate $Q$ over each macrocell; namely,

$$
\tilde{Q}_{n, \tilde{n}}(x)=\frac{\tilde{n}}{n} \sum_{s \in \mathcal{X}_{j_{1}, j_{2}}} Q(s) \quad \text { for all } \quad x \in \mathcal{X}_{j_{1}, j_{2}},
$$

Clearly, $\tilde{Q}_{n, \tilde{n}}$ is piecewise constant with respect to the partition of $\mathcal{X}$ into macrocells. The coarse-grained process $\tilde{Q}_{n, \tilde{n}}$ takes values in the space of macrostates $L^{2}(\mathcal{X})$.

In what follows we shall be interested in a double limit in which both $n \rightarrow \infty$ and $\tilde{n} \rightarrow \infty$, with $n / \tilde{n} \rightarrow \infty$. We refer to this double limit as the continuum limit. In order to deduce the limiting behavior of $\tilde{Q}_{n, \tilde{n}}$ under either the canonical ensemble (9) or the microcanonical ensemble (10), we first estabilish a basic theorem that describes its behavior with respect to $\Pi_{n}(d Q)$. The formulation of this theorem requires some definitions, which we now state.

Associated with the prior distribution $\rho$ is its cumulant generating function

$$
f(\eta)=\log \int_{R} \exp (\eta y) \rho(d y) \quad(\eta \in R) .
$$

In view of the decay condition (11), $f(\eta)$ is defined and continuous for all $\eta \in R$. Moreover, $f$ is convex function. The convex function $i$ conjugate to $f$, namely, the Legendre-Fenchel 
transform of $f$, is defined by

$$
i(y)=\sup _{\eta}[\eta y-f(\eta)] \quad(y \in R) .
$$

It is known that $i$ achieves its unique minimum value of 0 over $R$ at $\bar{y} \doteq \int y \rho(d y)$. The reader is referred to [12, 18] for these definitions and properties.

In terms of these standard constructions, we define the information functional

$$
I(q)=\int_{\mathcal{X}} i(q(x)) d x \quad\left(q \in L^{2}(\mathcal{X})\right) .
$$

In the terminology of large deviation theory, $I$ is a convex rate function; that is, it is a convex, lower semi-continuous functional mapping $L^{2}(\mathcal{X})$ into the extended interval $[0,+\infty]$. In fact, the information functional $I$ is the rate function for the basic large deviation principle satisfied by the coarse-grained process $\tilde{Q}_{n, \tilde{n}}$ with respect to the product prior distribution $\Pi_{n}(d Q)$. In the following theorem we state a simplified version of this large deviation principle. In another paper [21], we state and prove the general version.

Theorem 1. For any Borel subset $B$ of $L^{2}(\mathcal{X})$ that is a continuity set for the rate function $I$, the following double limit holds:

$$
\lim _{\tilde{n} \rightarrow \infty} \lim _{n \rightarrow \infty} \frac{1}{n} \log \prod_{n}\left\{\tilde{Q}_{n, \tilde{n}} \in B\right\}=-I(B),
$$

where $I(B) \doteq \inf \{I(q): q \in B\}$.

Here, we use the notion of a continuity set $B$ for $I$ to assert simply a double limit rather than the standard pair of large deviation upper and lower bounds for closed sets $B$ and open sets $B$, respectively. By a continuity set for the information functional (15) we mean any Borel set $B \subset L^{2}(\mathcal{X})$ with the property that $I\left(B^{0}\right)=I(\bar{B})$, where $B^{0}$ is the interior of $B$ and $\bar{B}$ is the closure of $B$. Under suitable conditions on $\rho$, such as (11), the continuity sets of $I$ are rich enough to encompass the sets that arise in practical applications of the result. The double limit (16) then conveys conceptually the content of the rigorous large deviation principle given in [21]. The proof relies essentially on the classical Cramér theorem for sample means of independent and identically distributed random variables 12, 18]. Roughly speaking, the theorem follows by applying Cramér's theorem to the local average that defines the coarse-grained process $\tilde{Q}_{n, \tilde{n}}$ over each macrocell $\mathcal{X}_{j_{1}, j_{2}}$, and then by integrating the results for each macrocell over the entire domain $\mathcal{X}$.

The asymptotic formula (16) give an exponential-order corrections to the law of large numbers behavior of the coarse-grained process $\tilde{Q}_{n, \tilde{n}}$. That is, finite departures of $\tilde{Q}_{n, \tilde{n}}$ from its mean value, the constant $\bar{y} \doteq \int y \rho(d y)$, have exponentially small probability as $n \rightarrow \infty$. If we take $B=\left\{q \in L^{2}(\mathcal{X}):\|q-\bar{y}\| \geq \delta>0\right\}$ in (16), then we have, for large $n$, $\tilde{n}$ and $n / \tilde{n}$,

$$
\Pi_{n}\left\{\tilde{Q}_{n, \tilde{n}} \in B\right\} \leq e^{-n I(B) / 2}
$$


in the formula $I(B)>0$ for any finite $\delta$, while $I(\bar{y})=0$.

We may summarize the content of Theorem 1 in the formal asymptotic statement that, for any macrostate $q \in L^{2}(\mathcal{X})$,

$$
\Pi_{n}\left\{\tilde{Q}_{n, \tilde{n}} \approx q\right\} \sim e^{-n I(q)} \quad \text { in the continuum limit. }
$$

Here, the symbol $\approx$ means close in the strong topology of $L^{2}(\mathcal{X})$. The equivalence between this formal statement and the precise result (16) can be seen by using balls $B_{r}(q)$ of arbitrarily small radius $r$ centered at $q$, and the fact that $I(q)=\lim _{r \rightarrow 0} I\left(B_{r}(q)\right)$. This asymptotic expression also provides the heuristic interpretation of the rate functional $I$ as a negative entropy. Indeed, $-I(q)$ quantifies the multiplicity of the microstates that correspond under the coarse-graining to a macrostate $q$. Equivalently, $I(q)$ represents the information lost in going from the microscopic to the macroscopic level of description.

3.2 Canonical model. We now turn to the analysis of the statistical equilibrium model governed by the canonical ensemble (9). The following theorem characterizes the continuum limit for that model, using the asymptotics for the coarse-grained process $\tilde{Q}_{n, \tilde{n}}$.

Theorem 2. With respect to the canonical ensemble $P_{n, \beta, \gamma}(d Q)$, the coarse-grained process $\tilde{Q}_{n, \tilde{n}}$ satisfies the double limit

$$
\lim _{\tilde{n} \rightarrow \infty} \lim _{n \rightarrow \infty} \frac{1}{n} \log P_{n, \beta, \gamma}\left\{\tilde{Q}_{n, \tilde{n}} \in B\right\}=-I_{\beta, \gamma}(B),
$$

for any Borel subset $B$ of $L^{2}(\mathcal{X})$ that is a continuity set for $I_{\beta, \gamma}$; in this formula,

$$
I_{\beta, \gamma}(q) \doteq I(q)+\beta H(q)+\gamma C(q)-\Phi(\beta, \gamma),
$$

where

$$
\begin{aligned}
\Phi(\beta, \gamma) & \doteq \min _{q \in L^{2}(\mathcal{X})}[I(q)+\beta H(q)+\gamma C(q)] \\
& =-\lim _{n \rightarrow \infty} \frac{1}{n} \log Z_{n}(\beta, \gamma) .
\end{aligned}
$$

The proof of this theorem is indicated in our companion paper [20]. The key idea is to represent the interaction functions $H_{n}$ and $C_{n}$ in the Gibbs measure (9) in terms of the coarse-grained process and the corresponding continuum functionals $H$ and $C$. This representation is provided by the following approximations

$$
H_{n}(Q)=H\left(\tilde{Q}_{n, \tilde{n}}\right)+o(1), \quad C_{n}(Q)=C\left(\tilde{Q}_{n, \tilde{n}}\right)+o(1),
$$

in which the $o(1)$ errors are uniformly small over $Q \in \Omega_{n}$. Here, and henceforth, we evaluate the functionals $H$ and $C$ defined in (田) and (5) on macrostates $q \in L^{2}(\mathcal{X})$. The 
streamfunction $\psi$ corresponding to any such $q$ is the solution to $-\Delta \psi+r^{-2} \psi+b=q$ in $\mathcal{X}$ with appropriate boundary conditions on $\partial \mathcal{X}$; that is, $\psi=G(q-b)$, where $G$ denotes the Green operator for $-\Delta+r^{-2}$ :

$$
G z(x)=\int_{\mathcal{X}} g\left(x, x^{\prime}\right) z\left(x^{\prime}\right) d x^{\prime} \quad\left(z \in L^{2}(\mathcal{X})\right) .
$$

The approximations (21) express the fundamental fact that the global invariants $H$ and $C$ are not sensitive to the small-scale fluctuations of the microstate $Q$, being almost unchanged by the local averaging that defines the coarse-grained process. The quadratic self-interaction term in $H$ has this property because it is defined by the long-range interaction function $g\left(x, x^{\prime}\right)$. C and the term in $H$ arising from interaction with the topography have this property because they are linear (affine). With the representations in hand, the large deviation limit (18) and the limit in (20) can be established by general methods, namely, the Laplace method for the asymptotics of large deviation-type expectations. As the proofs are very similar to those already given in [5], we omit them here.

¿From the point of view of predicting the coherent states in a turbulent fluid, the essential content of the large deviation principle for the canonical ensemble lies in the canonical information functional $I_{\beta, \gamma}$. According to (18), the most probable macrostates $q$ are those at which $I_{\beta, \gamma}(q)$ achieves its minimum value of 0 . For this reason, we define the set of equilibrium states associated with given canonical parameters $\beta, \gamma \in R$ to be

$$
\mathcal{E}_{\beta, \gamma} \doteq\left\{q \in L^{2}(\mathcal{X}): I_{\beta, \gamma}(q)=0\right\}=\arg \min [I+\beta H+\gamma C] .
$$

Any macrostate $q$ that does not lie in $\mathcal{E}_{\beta, \gamma}$ has an exponentially small probability of being observed as a coarse-grained state in the continuum limit; indeed, for such a macrostate $I_{\beta, \gamma}(q) \geq \delta$ for some positive $\delta$, and therefore the large deviation principle implies that for large $n$ and $\tilde{n}$

$$
P_{n, \beta, \gamma}\left\{\tilde{Q}_{n, \tilde{n}} \approx q\right\} \sim e^{-n I_{\beta, \gamma}(q)} \leq e^{-n \delta} .
$$

In light of this sharp estimate, we see that the equilibrium macrostates in $\mathcal{E}_{\beta, \gamma}$ are overwhelmingly most probable among all possible coarse-grained states of the turbulent system. Consequently, the main predictions of the statistical equilibrium theory in its canonical form are derived by solving the unconstrained minimization problem whose objective functional is $I+\beta H+\gamma C$. The existence of at least one equilibrium state $\bar{q}$ in $\mathcal{E}_{\beta, \gamma}$ for each given $\beta, \gamma \in R$ can be deduced readily by the direct methods of the calculus of variations. In general, $\mathcal{E}_{\beta, \gamma}$ may contain more than one macrostate, in which case the statistical equilbrium model exhibits a phase transition.

Let us now display the first-order conditions for the variational problem whose solutions are the equilibrium states in the canonical model. At a given solution $\bar{q} \in \mathcal{E}_{\beta, \gamma}$, there holds

$$
\begin{aligned}
0 & =\delta(I+\beta H+\gamma C)(\bar{q}) \\
& =\int_{\mathcal{X}}\left[i^{\prime}(\bar{q})+\beta \bar{\psi}+\gamma\right] \delta q d x
\end{aligned}
$$


where $\bar{\psi}$ is the streamfunction corresponding to $\bar{q}$, and $\delta q$ denotes a variation in $L^{2}(\mathcal{X})$. From this calculation we obtain the equilibrium equation $i^{\prime}(\bar{q})=-\beta \bar{\psi}-\gamma$, which we can express in the form

$$
\bar{q}=-\Delta \bar{\psi}+r^{-2} \bar{\psi}+b=f^{\prime}(-\beta \bar{\psi}-\gamma) .
$$

The last expression uses the fact that, since $f$ and $i$ are conjugate convex functions, their first derivatives $f^{\prime}$ and $i^{\prime}$ are inverse functions. Thus, the statistical equilibrium model produces a semilinear elliptic equation for the streamfunction $\bar{\psi}$ of the most probable flow. We shall refer to (25) as the mean-field equation. The predicted dependence $f^{\prime}$ of the mean potential vorticity on the mean streamfunction is determined solely by the statistical properties of the small-scale fluctuations in the model, since the prior distribution $\rho$ determines $f$ through $(13)$. With a fixed prior distribution $\rho$, the branches of most probable, or coherent, states are parametrized by $\beta$ and $\gamma$, which enter nonlinearly in (25). The mean-field equation can possess nonunique solutions, and its solutions branches can bifurcate.

Let us also record the second-order conditions at an equilibrium state $\bar{q}$. With $\delta \psi$ denoting the solution to $\left(-\Delta+r^{-2}\right) \delta \psi=\delta q$ under appropriate boundary conditions, there holds

$$
\begin{aligned}
0 & \leq \delta^{2}(I+\beta H+\gamma C)(\bar{q}) \\
& =\int_{\mathcal{X}}\left\{i^{\prime \prime}(\bar{q})(\delta q)^{2}+\beta\left[\left(\frac{\partial \delta \psi}{\partial x_{1}}\right)^{2}+\left(\frac{\partial \delta \psi}{\partial x_{2}}\right)^{2}+r^{-2}(\delta \psi)^{2}\right]\right\} d x
\end{aligned}
$$

This condition is equivalent to the nonnegative-definiteness of the bounded, symmetric operator $i^{\prime \prime}(\bar{q})+\beta G$ on $L^{2}(\mathcal{X})$, where $i^{\prime \prime}(\bar{q})$ is a multiplication operator and $G$ is the Green operator $(22)$. Both of these component operators are positive-definite. Consequently, the second-order conditions are automatically satisfied whenever $\beta$ is positive. When $\beta$ is negative, however, a critical point $\bar{q}$ satisfying the mean-field equations is not an equilibrium state unless the second variation of $I+\beta H$ is nonnegative-definite at $\bar{q}$. Accordingly, the second-order conditions are crucial in the negative temperature regime, which is often the regime of most interest in the study of isolated coherent structures. Finally, we note that if the second variation is strictly positive-definite at $\bar{q}$, then $\mathcal{E}_{\beta, \gamma}=\{\bar{q}\}$, and the equilibrium is isolated and nondegenerate. Conversely, the degeneracy of the second variation signals the presence of a phase transition.

3.3 Microcanonical model. In some respects the microcanonical ensemble (10) defines a more natural model than the corresponding canonical ensemble. From a physical point of view, the canonical parametrization of equilibrium states by an inverse temperature $\beta$ and a chemical potential $\gamma$ is undesirable because the coherent structures are not maintained by contact with a bath having these parameters. Rather, the equilibrium states represent organized flows on the large scales which contain the energy $E$ and circulation $\Gamma$ and are isolated from the turbulent fluctuations on the small-scales. It is therefore reasonable to 
parametrize such flows by $E$ and $\Gamma$. From a mathematical standpoint, we are compelled to study the microcanonical formulation of the statistical equilibrium theory by virtue of the fact, which we establish in Section 4, that the microcanonical model is not always equivalent to the canonical model in the continuum limit.

The following theorem characterizes the continuum limit for the microcanonical model in terms of the coarse-grained process $\tilde{Q}_{n, \tilde{n}}$.

Theorem 3. With respect to the microcanonical ensemble $P_{n}^{E, \Gamma}(d Q)$, the coarse-grained process $\tilde{Q}_{n, \tilde{n}}$ satisfies the double limit

$$
\lim _{\tilde{n} \rightarrow \infty} \lim _{n \rightarrow \infty} \frac{1}{n} \log P_{n}^{E, \Gamma}\left\{\tilde{Q}_{n, \tilde{n}} \in B\right\}=-I^{E, \Gamma}(B)
$$

for any Borel subset $B$ of $L^{2}(\mathcal{X})$ that is a continuity set for $I^{E, \Gamma}$; in this formula,

$$
I^{E, \Gamma}(q) \doteq \begin{cases}I(q)+S(E, \Gamma) & \text { if } H(q)=E, C(q)=\Gamma \\ +\infty & \text { otherwise, }\end{cases}
$$

where

$$
\begin{aligned}
S(E, \Gamma) & \doteq-\min \{I(q): H(q)=E, C(q)=\Gamma\} \\
& =\lim _{n \rightarrow \infty} \frac{1}{n} \log \Pi_{n}\left\{H_{n}=E, C_{n}=\Gamma\right\}
\end{aligned}
$$

We reiterate our earlier remark that we have taken the microcanonical constraints to be exact equalities for the sake of clarity in the exposition. To obtain mathematically rigorous versions of these results, we first replace the microcanonical constraints by the containments $H_{n} \in[E-\epsilon, E+\epsilon]$ and $C_{n} \in[\Gamma-\delta, \Gamma+\delta]$ with finite $\epsilon, \delta>0$, and we then take a third limit as $\epsilon, \delta \rightarrow 0$ in (27) after the limits on $n$ and $\tilde{n}$.

This theorem is a simplified version of a general theorem that we formulate and prove in our companion paper [20]. As in the analysis of canonical model, the representations (21) are fundamental to the proof. With these approximations and the basic large deviation principle (16) in hand, the large deviation principle (27) can be deduced directly from the general results in 20$]$.

The large deviation principle for the microcanonical ensemble involves the microcanonical information functional $I^{E, \Gamma}$. Among the macrostates lying on the microcanonical manifold $H=E, C=\Gamma$, the most probable macrostates $q$ are those at which $I^{E, \Gamma}$ achieves its minimum value of 0 . These macrostates compose the set of equilibrium states associated with given microcanonical parameters $E>0, \Gamma \in R$; namely,

$$
\mathcal{E}^{E, \Gamma} \doteq\left\{q \in L^{2}(\mathcal{X}): I^{E, \Gamma}(q)=0\right\}=\arg \min \{I: H=E, C=\Gamma\}
$$


As in the canonical model, any macrostate $q$ that does not lie in the equilibrium set has an exponentially small probability of being observed as a coarse-grained state in the continuum limit. Conversely, the equilibrium macrostates in $\mathcal{E}^{E, \Gamma}$, which solve the constrained minimization problem with objective functional $I$, are the overwhelmingly most probable coarse-grained states compatible with the microcanonical constraints $H=E, C=\Gamma$. Again, as in the canonical model, the existence of an equilibrium state $\bar{q}$ in $\mathcal{E}^{E, \Gamma}$ is ensured by direct methods. Since the equality constraint $H=E$ makes the microcanonical manifold a nonconvex set, constrained minimizers may be nonunique, and hence $\mathcal{E}^{E, \Gamma}$ may contain multiple equilibrium macrostates.

The first-order conditions for a microcanonical equilibrium $\bar{q} \in \mathcal{E}^{E, \Gamma}$ are identical to (24), except that $\beta$ and $\gamma$ are Lagrange multipliers for the energy and circulation constraints, respectively. The solution triple $(\bar{q}, \beta, \gamma)$ is determined, in principle, by the given constraint pair $(E, \Gamma)$, since the multipliers are uniquely determined by the critical point $\bar{q}$. Similarly, the mean-field equation (25) holds without change in the microcanonical model, except that the parameters $\beta$ and $\gamma$ appearing in it are also unknowns.

The second-order conditions, on the other hand, are fundamentally altered by shifting from the canonical to microcanonical formulation. ¿From general principles in optimization, we know that the nonnegativity condition (26) at a constrained minimizer $\bar{q}$ holds for all variations $\delta q$ that are infinitesimally compatible with the constraints, but not necessarily for arbitrary variations $\delta q$ [27, 51]. Thus, we find that the second-order conditions appropriate to a macrostate $\bar{q} \in \mathcal{E}^{E, \Gamma}$ are that (26) holds for all $\delta q$ satisfying the linearized sideconditions

$$
\delta H(\bar{q})=\int_{\mathcal{X}} \bar{\psi} \delta q d x=0 \quad \text { and } \quad \delta C(\bar{q})=\int_{\mathcal{X}} \delta q d x=0
$$

Given this characterization of the constrained minimizers of $I$ subject to $H=E$ and $C=\Gamma$, we see that set of microcanonical equilibria is potentially larger than the corresponding set of canonical equilibria.

This difference between the canonical and microcanonical equilibrium equations at second-order underlies all of our subsequent development. Broadly speaking, it implies that families of microcanonical equilibria are richer than corresponding families of canonical equilibria, and that nonlinear stability criteria based on the microcanonical formulation are finer than corresponding criteria for the canonical formulation.

\section{Equivalence and nonequivalence}

We now turn our attention to the relation between the equilibrium sets $\mathcal{E}_{\beta, \gamma}$ for the canonical model and the equilibrium sets $\mathcal{E}^{E, \Gamma}$ for the microcanonical model. In most statistical equilibrium models, the canonical and microcanonical ensembles are equivalent, in the sense that there is a one-to-one correspondence between their equilibrium states. For the local 
mean-field models of coherent structures in turbulence, however, there can be microcanonical equilibria that cannot be realized as canonical equilibria. Moreover, these equilibrium states are neither rare nor pathological. Rather, they are often the coherent mean flows of greatest physical interest. In the analysis to follow, we show how the properties of the thermodynamic functions in the microcanonical and canonical models determine the correspondence, or lack of correspondence, between equilibria for these two models.

4.1 Thermodynamic functions. The fundamental thermodynamic function for the microcanonical model is the value function $S(E, \Gamma)$ in the constrained maximum entropy principle (29) whose solutions constitute the equilibrium set $\mathcal{E}^{E, \Gamma}$. Similarly, the fundamental thermodynamic function for the canonical model is the value function $\Phi(\beta, \gamma)$ in the free maximum entropy principle (20) whose solutions constitute the equilibrium set $\mathcal{E}_{\beta, \gamma}$. These two functions are conjugate functions in the sense of convex analysis [27, 51]; that is, they are related by the identity

$$
\Phi(\beta, \gamma)=\inf _{E, \Gamma}[\beta E+\gamma \Gamma-S(E, \Gamma)]
$$

The proof simply amounts to writing the free minimization in $(20)$ in terms of the constrained minization in (29):

$$
\begin{aligned}
\min _{q}[I+\beta H+\gamma \Gamma] & =\inf _{E, \Gamma} \min _{q}\{I+\beta H+\gamma \Gamma: H=E, C=\Gamma\} \\
& =\inf _{E, \Gamma}[\beta E+\gamma \Gamma-S(E, \Gamma)] .
\end{aligned}
$$

In other words, $\Phi=S^{*}$ is the Legendre-Fenchel transform of $S$. Consequently, $\Phi$ is a concave function of $(\beta, \gamma)$, which runs over $R^{2}$. By contrast, $S$ itself is not necessarily concave. The concave hull of $S$ is furnished by the conjugate function of $\Phi$, namely, $\Phi^{*}=S^{* *}$, which satisfies the inequality

$$
S(E, \Gamma) \leq \inf _{\beta, \gamma}[\beta E+\gamma \Gamma-\Phi(\beta, \gamma)]=S^{* *}(E, \Gamma)
$$

The relation between microcanonical equilibria and canonical equilibria depends crucially on the concavity properties of the microcanonical entropy $S$. Henceforth, we shall consider the function $S$ to be defined on a domain $\mathcal{A}$, which we take to be the largest open subset of $R^{2}$ consisting of admissible constraint pairs $(E, \Gamma)$ for the microcanonical model; such a constraint pair is admissible if $(E, \Gamma)=(H(q), C(q))$ for some $q \in L^{2}(\mathcal{X})$ with $I(q)<+\infty$. We call this domain $\mathcal{A}$ the admissible set for the microcanonical model. Since, in general, $S$ is not a concave function on $\mathcal{A}$, we introduce the subset $\mathcal{C} \subseteq \mathcal{A}$ on which the concave hull $S^{* *}$ coincides with $S$; that is, $(E, \Gamma) \in \mathcal{C}$ if and only if $S^{* *}(E, \Gamma)=S(E, \Gamma)$. There is another equivalent definition of $\mathcal{C}$. Namely, $\mathcal{C}$ consists of those points $(E, \Gamma) \in \mathcal{A}$ for which there exists some $(\beta, \gamma) \in R^{2}$ such that

$$
S\left(E^{\prime}, \Gamma^{\prime}\right) \leq S(E, \Gamma)+\beta\left(E^{\prime}-E\right)+\gamma\left(\Gamma^{\prime}-\Gamma\right)
$$


for all $\left(E^{\prime}, \Gamma^{\prime}\right) \in \mathcal{A}$. This condition means that $S$ has a supporting plane, with normal determined by $(\beta, \gamma)$, at the point $(E, \Gamma)$. Such points $(E, \Gamma)$ are precisely those points of $\mathcal{A}$ at which $S$ has a (nonempty) superdifferential, which is the set of all $(\beta, \gamma)$ for which the above condition holds [27, 51].

4.2 Microcanonical and canonical equilibrium sets. The set $\mathcal{C}$, which we call the concavity set, plays a pivotal role in the criteria for equivalence of ensembles. The following theorem gives results of this kind.

\section{Theorem 4.}

(a) If $(E, \Gamma) \in \mathcal{A}$ belongs to $\mathcal{C}$, then $\mathcal{E}^{E, \Gamma} \subseteq \mathcal{E}_{\beta, \gamma}$ for some $(\beta, \gamma)$.

(b) If $(E, \Gamma) \in \mathcal{A}$ does not belong to $\mathcal{C}$, then $\mathcal{E}^{E, \Gamma} \cap \mathcal{E}_{\beta, \gamma}=\emptyset$ for all $(\beta, \gamma)$.

Proof. (a) The hypothesis means that equality holds in (33) and is attained at some $(\beta, \gamma)$ for which

$$
S(E, \Gamma)=\beta E+\gamma \Gamma-\Phi(\beta, \gamma) .
$$

To show the claimed containment, take any $\bar{q} \in \mathcal{E}^{E, \Gamma}$ and note that $H(\bar{q})=E, C(\bar{q})=\Gamma$ and $I(\bar{q})=-S(E, \Gamma)$. Substitution of these expressions into the above equality yields

$$
\begin{aligned}
I(\bar{q})+\beta H(\bar{q})+\gamma C(\bar{q}) & =-S(E, \Gamma)+\beta E+\gamma \Gamma \\
& =\Phi(\beta, \gamma)=\min _{q}[I(q)+\beta H(q)+\gamma C(q)],
\end{aligned}
$$

using (20). Since $\mathcal{E}_{\beta, \gamma}$ consists of the minimizers of $I+\beta H+\gamma C$, it follows that $\bar{q} \in \mathcal{E}_{\beta, \gamma}$. This completes the proof of (a).

(b) A complementary argument to that used in (a) applies. Now, the hypothesis means that, for all $(\beta, \gamma)$,

$$
S(E, \Gamma)<\beta E+\gamma \Gamma-\Phi(\beta, \gamma)
$$

Then, any $\bar{q} \in \mathcal{E}^{E, \Gamma}$ satisfies

$$
\begin{aligned}
I(\bar{q})+\beta H(\bar{q})+\gamma C(\bar{q}) & =-S(E, \Gamma)+\beta E+\gamma \Gamma \\
& >\Phi(\beta, \gamma)=\min _{q}[I(q)+\beta H(q)+\gamma C(q)] .
\end{aligned}
$$

Thus, $\bar{q}$ does not minimize $I+\beta H+\gamma C$, and hence does not belong to $\mathcal{E}_{\beta, \gamma}$. Since $(\beta, \gamma)$ is arbitrary, this completes the proof of (b).

¿From Theorem 4 we see that, for constraint pairs in $\mathcal{C}$, the microcanonical equilibria are contained in a corresponding canonical equilibrium set, while, for constraint pairs in $\mathcal{A} \backslash \mathcal{C}$, the microcanonical equilibria are not contained in any canonical equilibrium set. Consequently, whenever $\mathcal{C} \neq \mathcal{A}$ the canonical equilibria do not exhaust the admissible microcanonical constraint pairs, and there are microcanonical equilibria that are not realized by any canonical equilibria. On the other hand, all canonical equilibria are contained in 
some microcanonical equilibrium set, and $\mathcal{C}$ is exhausted by the constraint pairs realized by all canonical equilibria. These further results are given in the following theorem.

\section{Theorem 5 .}

(a) The concavity set $\mathcal{C}$ consists of all constraint pairs realized by the canonical equilibria; that is,

$$
\mathcal{C}=\bigcup\left\{(H, C)\left(\mathcal{E}_{\beta, \gamma}\right):(\beta, \gamma) \in R^{2}\right\}
$$

(b) Each canonical equilibrium set $\mathcal{E}_{\beta, \gamma}$ consists of all microcanonical equilibria whose constraint pairs are realized by $\mathcal{E}_{\beta, \gamma}$; that is, for any $(\beta, \gamma)$,

$$
\mathcal{E}_{\beta, \gamma}=\bigcup\left\{\mathcal{E}^{E, \Gamma}:(E, \Gamma) \in(H, C)\left(\mathcal{E}_{\beta, \gamma}\right)\right\}
$$

Proof. (a) The containment of $\mathcal{C}$ in the union is immediate from Theorem 4a. To show the opposite containment we argue by contradiction, supposing that for some $(\beta, \gamma)$ and some $\bar{q} \in \mathcal{E}_{\beta, \gamma},(E, \Gamma)=(H(\bar{q}), C(\bar{q})) \in \mathcal{A} \backslash \mathcal{C}$. Then, we find that

$$
\begin{aligned}
S(E, \Gamma) & <\beta E+\gamma \Gamma-\Phi(\beta, \gamma) \\
& =-I(\bar{q}) \leq S(E, \Gamma)
\end{aligned}
$$

using (20) and (29) as in the proof of Theorem 4b. We thus obtain the desired contradiction. This completes the proof of (a).

(b) The containment of $\mathcal{E}_{\beta, \gamma}$ in the union is straightforward. Let $\bar{q} \in \mathcal{E}_{\beta, \gamma}$, and set $E=H(\bar{q})$ and $\Gamma=C(\bar{q})$. Then, $I(\bar{q})+\beta E+\gamma \Gamma \leq I(q)+\beta H(q)+\gamma C(q)$ for all $q$. For those $q$ which satisfy the constraints $H(q)=E, C(q)=\Gamma$, we therefore find that $I(\bar{q}) \leq I(q)$. Hence, $\bar{q} \in \mathcal{E}^{E, \Gamma}$.

The opposite containment is also straightforward. If $E=H(\tilde{q})$ and $\Gamma=C(\tilde{q})$ for some $\tilde{q} \in \mathcal{E}_{\beta, \gamma}$, then for any $\bar{q} \in \mathcal{E}^{E, \Gamma}$, we have $I(\bar{q}) \leq I(\tilde{q})$. Since $\tilde{q} \in \mathcal{E}_{\beta, \gamma}$, we obtain

$$
\begin{aligned}
\min _{q}[I(q)+\beta H(q)+\gamma C(q)] & =I(\tilde{q})+\beta E+\gamma \Gamma \\
& \geq I(\bar{q})+\beta H(\bar{q})+\gamma C(\bar{q}) .
\end{aligned}
$$

Hence, $\bar{q} \in \mathcal{E}_{\beta, \gamma}$. This completes the proof of (b).

Theorems 4 and 5 allow us to classify the microcanonical constraint parameters $(E, \Gamma)$ according to whether or not equivalence of ensembles holds for those parameters. In fact, the admissible set $\mathcal{A}$ can be decomposed into three disjoint sets, where (1) there is a one-to-one correspondence between microcanonical and canonical equilibria, (2) there is a many-to-one correspondence from microcanonical equlibria to canonical equilibria, and (3) there is no correspondence. In order to simplify the precise statement of this result, let us assume that the microcanonical entropy $S(E, \Gamma)$ is differentiable on its domain $\mathcal{A}$. 
Then, for each microcanonical parameter $(E, \Gamma) \in \mathcal{A}$ there is a corresponding canonical parameter $(\beta, \gamma)$ determined locally by

$$
\beta=\frac{\partial S}{\partial E}, \quad \gamma=\frac{\partial S}{\partial \Gamma}
$$

Under this assumption, we have the following classification.

1. Full equivalence. If $(E, \Gamma)$ belongs to $\mathcal{C}$ and there is a unique point of contact between $S$ and its supporting plane at $(E, \Gamma)$, then $\mathcal{E}^{E, \Gamma}$ coincides with $\mathcal{E}_{\beta, \gamma}$

2. Partial equivalence. If $(E, \Gamma)$ belongs to $\mathcal{C}$ but there is more than one point of contact between $S$ and its supporting plane at $(E, \Gamma)$, then $\mathcal{E}^{E, \Gamma}$ is a strict subset of $\mathcal{E}_{\beta, \gamma}$. Moreover, $\mathcal{E}_{\beta, \gamma}$ contains all those $\mathcal{E}^{E^{\prime}, \Gamma^{\prime}}$ for which $\left(E^{\prime}, \Gamma^{\prime}\right)$ is also a point of contact.

3. Nonequivalence. If $(E, \Gamma)$ does not belonging to $\mathcal{C}$, then $\mathcal{E}^{E, \Gamma}$ is disjoint from $\mathcal{E}_{\beta, \gamma}$. In fact, $\mathcal{E}^{E, \Gamma}$ is disjoint from all canonical equilibrium sets.

The proofs of these results can be constructed easily using the same techniques as in the proofs of Theorems 4 and 5 . We therefore leave the necessary demonstrations to the reader. We give a complete discussion of these results in a more general setting in our paper [20], where we state and prove the corresponding results without the simplifying assumption that $S$ is differentiable. Experience with numerical solutions of these variational problems of this kind, however, strongly suggests that the differentiability assumption is essentially always satisfied. These computations also show that the parameter regime of nonequivalence can be quite wide and can contain many physically interesting equilibrium flows. In Section 6 , we present a computed example that illustrates this behavior.

\section{$5 \quad$ Nonlinear stability}

In either the canonical or the microcanonical model, the equilibrium macrostates determine steady mean flows that are the most probable flows compatible with the given parameters of the model. This statistical property of the mean flows can be interpreted as a stability property in a weak sense. That is, while the underlying ergodic dynamics continually produces unsteady perturbations in the microstate, the coarse-grained macrostate remains near the mean flow with very high probability. In other words, the construction of the steady mean flows as statistical equilibrium macrostates guarantees that they are stable with respect to perturbations on the microscopic scales. We now inquire whether these steady mean states are also stable in a strong sense with respect to macroscopic perturbations. Precisely, we investigate the evolution under ideal dynamics of any perturbed macroscopic state $q(t)$ that initially lies within a small, finite distance $\|q(0)-\bar{q}\|$ in $L^{2}(\mathcal{X})$ of an equilibrium macrostate $\bar{q}$.

In the canonical model, we find that the most probable state $\bar{q}$ for any $\beta$ and $\gamma$ satisfies the celebrated Arnold stability criteria, the canonical information functional $I_{\beta, \gamma}$ being 
the required Lyapunov functional. We collect these results in Subsection 5.1. In the microcanonical model, on the other hand, we encounter a gap in the classical stability criteria in the sense that there are microcanonical equilibria which are stable, but for which $I_{\beta, \gamma}$ does not satisfy the conditions needed in the Lyapunov stability argument. In Subsection 5.2, we therefore devise a more refined argument based on a penalization of this functional and thereby fill the gap in the known stability theorems.

5.1 Arnold stability theorems. The equilibria for the canonical model correspond to steady flows that satisfy the nonlinear stability criteria of Arnold [1], 33]. In this subsection we reformulate these classical results in the context of the statistical equilibrium theory.

Throughout this discussion we assume that for given values of the canonical parameters $\beta$ and $\gamma$, the equilibrium state $\bar{q} \in \mathcal{E}_{\beta, \gamma}$ is an isolated, nondegenerate minimizer of canonical information functional $I_{\beta, \gamma}$; otherwise, the stability of a single equilibrium state $\bar{q}$ cannot be expected. The fact that $\bar{q}$ is a minimizer of $I_{\beta, \gamma}$ over $L^{2}(\mathcal{X})$ guarantees that the second variation $\delta^{2} I_{\beta, \gamma}(\bar{q})$ appearing in (26) is nonnegative definite. A sufficient condition for $\bar{q}$ to be a nondegenerate minimizer is that $\delta^{2} I_{\beta, \gamma}(\bar{q})$ be strictly positive definite. More precisely, we say that $\bar{q}$ is an nondegenerate canonical equilibrium state if

$$
\mu \int_{\mathcal{X}}(\delta q)^{2} d x \leq \delta^{2} I_{\beta, \gamma}(\bar{q})
$$

for all variations $\delta q \in L^{2}(\mathcal{X})$, with a positive constant $\mu$ independent of $\delta q$. The optimal constant $\mu$ in (37) is the smallest eigenvalue of the operator $i^{\prime \prime}(\bar{q})+\beta G$, where $G$ is the Green operator (22). This fact is immediate from the identity

$$
\delta^{2} I_{\beta, \gamma}(\bar{q})=\delta^{2}(I+\beta H+\gamma \Gamma)(\bar{q})=\int_{\mathcal{X}}\left[i^{\prime \prime}(\bar{q})(\delta q)^{2}+\beta \delta q G \delta q\right] d x .
$$

An upper bound that complements the lower bound (37) also holds, namely,

$$
\delta^{2} I_{\beta, \gamma}(\bar{q}) \leq \nu \int_{\mathcal{X}}(\delta q)^{2} d x
$$

In this upper bound it suffices to take the constant $\nu=\max i^{\prime \prime}(\bar{q})+|\beta| / \lambda_{1}$, where $\lambda_{1}>0$ is the smallest eigenvalue of $-\Delta+r^{-2}$; the required bound on $i^{\prime \prime}(\bar{q})=1 / f^{\prime \prime}(-\beta \bar{\psi}-\gamma)$ follows easily from the fact that $f^{\prime \prime}(\eta)$ equals the variance of the distribution $z(\eta)^{-1} e^{\eta y} \rho(d y)$, which is bounded below by a positive constant uniformly for $\eta$ in a bounded interval.

The nonlinear stability result for the canonical model is summarized in the following theorem.

Theorem 6. If $\bar{q} \in \mathcal{E}_{\beta, \gamma}$ is a nondegenerate canonical equilibrium state, then the corresponding steady flow is stable; specifically, if $q(t)$ denotes the solution to (1) and if $\|q(0)-\bar{q}\|$ is sufficiently small, then for all time $t>0$

$$
\|q(t)-\bar{q}\| \leq c\|q(0)-\bar{q}\|
$$


for some finite constant $c$.

Proof. The proof relies on the fact that $I_{\beta, \gamma}$ is a conserved quantity for the dynamics (1). The conservation of $H$ and $C$ is immediate, since they are rugged invariants. The information functional $I$ is also an invariant under the ideal dynamics that governs $q(t)$, since it coincides with a certain generalized enstrophy integral (6) under the identification $a=i$. We claim that the invariant $I_{\beta, \gamma}$ satisfies

$$
\frac{\mu}{2}\|q-\bar{q}\|^{2} \leq I_{\beta, \gamma}(q) \leq 2 \nu\|q-\bar{q}\|^{2} .
$$

for all $q$ in a small $L^{2}$-neigborhood of $\bar{q}$. These estimates follow from the upper and lower bounds on the second variation $\delta^{2} I_{\beta, \gamma}(\bar{q})$ given in (37) and (37), in view of the fact that $I_{\beta, \gamma}(\bar{q})=0$ and $\delta I_{\beta, \gamma}(\bar{q})=0$. The derivation makes use of a standard estimation of the remainder terms in the second-order Taylor expansion of the smooth functional $I_{\beta, \gamma}$ about $\bar{q}$. Then, the usual Lyapunov argument yields

$$
\begin{aligned}
\frac{\mu}{2}\|q(t)-\bar{q}\|^{2} & \leq I_{\beta, \gamma}(q(t)) \\
& =I_{\beta, \gamma}(q(0)) \leq 2 \nu\|q(0)-\bar{q}\|^{2}
\end{aligned}
$$

for all $t>0$, thereby proving the theorem.

We remark that this proof of Lyapunov stability requires only that $I_{\beta, \gamma}(q(t)) \leq I_{\beta, \gamma}(q(0))$ for $t>0$. This observation allows us to make a connection with the Turkington model 49], which is based on an argument that only inequalities on convex generalized enstrophies constrain the ideal dynamics. Even though $I$ is treated as a fragile invariant in that framework, the nonlinear stability of $\bar{q}$ remains valid, since $H$ and $C$ are rugged invariants.

In the context of the statistical equilibrium theory, the classical stability criteria amount to sufficient conditions for the nondegeneracy of the minimizer $\bar{q}$. For positive temperature states $(\beta \geq 0)$, the so-called first Arnold theorem applies, while for negative temperature states $(\beta<0)$, the so-called second Arnold theorem applies [1, 33]. In either case the sufficient condition for stability is that the bounded, symmetric operator $i^{\prime \prime}(\bar{q})+\beta G$ be positive definite. This form of the stability condition can be translated into the familiar form used in deterministic studies of steady flows by means of the formula

$$
\frac{d \bar{q}}{d \bar{\psi}}=-\frac{\beta}{i^{\prime \prime}(\bar{q})}
$$

which follows from the mean-field equation (25) and the fact that $f^{\prime}$ and $i^{\prime}$ are inverse functions. In this form, the first Arnold theorem applies when $d \bar{q} / d \bar{\psi}<0$, while the second Arnold theorem applies when $0<d \bar{q} / d \bar{\psi}<\lambda_{1}$, where $\lambda_{1}$ is the smallest eigenvalue of $-\Delta+r^{-2}$. If a deterministic steady flow corresponding to a potential vorticity field $\bar{q}$ is submitted to these stability criteria, there often are instances when neither the first nor the 
second theorem applies; these steady flows correspond to critical points for $I_{\beta, \gamma}$ at which its second-variation is negative in some direction. By constrast, any nondegenerate canonical equilibrium state $\bar{q}$ satisfies these criteria, the first when $\beta \geq 0$ and the second when $\beta<0$. Thus, apart from degeneracies such as occur at phase transitions, the statistical equilibrium theory always produces mean flows that are both steady and stable.

5.2 Refined stability theorems. When a microcanonical equilibrium $\bar{q} \in \mathcal{E}^{E, \Gamma}$ does not lie in any canonical equilibrium set $\mathcal{E}_{\beta, \gamma}$, the stability results of the preceding subsection do not apply. Nevertheless, every nondegenerate equilibrium state for the microcanonical model determines a stable flow, as we now show by giving a more refined nonlinear stability analysis.

Again, we assume that $\bar{q} \in \mathcal{E}^{E, \Gamma}$ is the isolated, nondegenerate minimizer of the microcanonical information $I$ at given microcanonical constraint values $E$ and $\Gamma$. In the microcanonical model, however, the second-order conditions at a constrained minimizer $\bar{q}$ are subject to side-conditions on $\delta q$, which are the linearization of the constraints $H=E, C=\Gamma$. Precisely, we say that $\bar{q}$ is a nondegenerate microcanonical equilibrium state if (37) holds for all $\delta q \in L^{2}(\mathcal{X})$ that satisfy the linearized constraints (31), with a positive constant $\mu$ independent of these $\delta q$. The complementary upper bound (38) also holds at the microcanonical equilibrium $\bar{q}$, with a constant $\nu$ determined as in the canonical model; in fact, the upper bound also holds for $\delta q$ not satisfying the side-conditions (31).

Our strategy for proving the stability of $\bar{q}$ is to construct a Lyapunov functional in the form

$$
\begin{gathered}
L_{\sigma, \tau}^{E, \Gamma}(q) \doteq I(q)+S(E, \Gamma)+\beta[H(q)-E]+\gamma[C(q)-\Gamma] \\
+\frac{\sigma}{2}[H(q)-E]^{2}+\frac{\tau}{2}[C(q)-\Gamma]^{2}
\end{gathered}
$$

where $\beta$ and $\gamma$ are the Lagrange multipliers for the energy and circulations constraints, respectively, and $\sigma$ and $\tau$ are sufficiently large positive constants. The terms in (39) scaled by $\sigma$ and $\tau$ penalize departures from the microcanonical constraints and thereby capture the microcanonical conditioning in the Lyapunov functional. Moreover, these terms do not change the value of the Lyapunov functional or its first variation at $\bar{q}$, which are

$$
L_{\sigma, \tau}^{E, \Gamma}(\bar{q})=0, \quad \delta L_{\sigma, \tau}^{E, \Gamma}(\bar{q})=\delta(I+\beta H+\gamma C)(\bar{q})=0 .
$$

For this reason, it is possible to choose finite constants $\sigma$ and $\tau$ so that $L_{\sigma, \tau}^{E, \Gamma}$ has a nondegenerate, unconstrained minimum at $\bar{q}$. In this sense $L_{\sigma, \tau}^{E, \Gamma}$ is identical to the "augmented Lagrangian" often used in numerical methods of constrained optimization «, 38].

In the case of full equivalence, when the concavity condition (34) holds as a strict inequality for all $\left(E^{\prime}, \Gamma^{\prime}\right) \neq(E, \Gamma)$, the penalizing terms are unnecessary, because $L_{0,0}^{E, \Gamma}$ coincides with $I_{\beta, \gamma}$ and hence furnishes a Lyapunov functional at $\bar{q}$. Indeed, the argument used to prove part (a) of Theorem 4 applies to this situation, and guarantees that $L_{0,0}^{E, \Gamma}(q)>$ 
$L_{0,0}^{E, \Gamma}(\bar{q})=0$ for all $q \neq \bar{q}$. In the cases of nonequivalence or partial equivalence, however, when the microcanonical equilibrium $\bar{q}$ may not be contained in the corresponding canonical equilibrium set, $L_{0,0}^{E, \Gamma}$ may not be a Lyapunov functional at $\bar{q}$. In those cases, $\delta^{2} L_{0,0}^{E, \Gamma}$ may be negative for variations $\delta q$ that are not tangential to the constraint manifold $H=E, C=\Gamma$. In general, it is therefore necessary to include penalization parameters $\sigma$ and $\tau$ so that $\delta^{2} L_{\sigma, \tau}^{E, \Gamma}(\bar{q})$ is positive definite. An explicit calculation of this second-variation, namely,

$$
\delta^{2} L_{\sigma, \tau}^{E, \Gamma}(\bar{q})=\delta^{2}(I+\beta H+\gamma C)(\bar{q})+\sigma\left\{\int_{\mathcal{X}} \bar{\psi} \delta q d x\right\}^{2}+\tau\left\{\int_{\mathcal{X}} \delta q d x\right\}^{2}
$$

suggests that it is indeed positive definite on arbitrary variations $\delta q$ when $\sigma$ and $\tau$ are sufficiently large.

The nonlinear stability result for the microcanonical model is the content of the following theorem.

Theorem 7. If $\bar{q} \in \mathcal{E}^{E, \Gamma}$ is a nondegenerate microcanonical equilibrium state, then the corresponding steady flow is stable; specifically, if $q(t)$ denotes the solution to (1) and if $\|q(0)-\bar{q}\|$ is sufficiently small, then for all time $t>0$

$$
\|q(t)-\bar{q}\| \leq c\|q(0)-\bar{q}\|
$$

for some finite constant $c$.

Proof. The crux of the proof is to demonstrate that the second variation of $L_{\sigma, \tau}^{E, \Gamma}(\bar{q})$ is strictly positive definite when $\sigma$ and $\tau$ as fixed large enough. This analysis makes use of the bilinear form associated with the operator $i^{\prime \prime}(\bar{q})+\beta G$, which we denote by

$$
D_{2}\left(z_{1}, z_{2}\right) \doteq \int_{\mathcal{X}}\left[i^{\prime \prime}(\bar{q}) z_{1} z_{2}+\beta z_{1} G z_{2}\right] d x \quad\left(z_{1}, z_{2} \in L^{2}(\mathcal{X})\right) .
$$

¿From the identity in (26), it is clear that $D_{2}(\delta q, \delta q)=\delta^{2}(I+\beta H+\gamma C)(\bar{q})$. Also, we let $\left(z_{1}, z_{2}\right)=\int z_{1} z_{2} d x$ denote the inner product on $L^{2}(\mathcal{X})$.

We decompose any variation $\delta q \in L^{2}(\mathcal{X})$ into a part $\delta q^{\|}$tangent to the microcanonical manifold at $\bar{q}$, and a part $\delta q^{\perp}$ orthogonal to it; that is,

$$
\delta q=\delta q^{\|}+\delta q^{\perp}
$$

where $\delta q^{\perp}=\xi \bar{\psi}+\eta 1$ for some $\xi, \eta \in R$, and $\left(\delta q^{\|}, \bar{\psi}\right)=0,\left(\delta q^{\|}, 1\right)=0$. It is easy to verify that the functions $\bar{\psi}$ and 1 are linearly independent, given that $E \neq 0$ in the microcanonical energy constraint. Thus, the components $\xi$ and $\eta$ are uniquely determined by $\delta q$, in that they solve the associated normal equations. A straightforward analysis then shows that the inequality

$$
\frac{(\bar{\psi}, \delta q)^{2}}{\|\bar{\psi}\|^{2}}+\frac{(1, \delta q)^{2}}{\|1\|^{2}}=\frac{\left(\bar{\psi}, \delta q^{\perp}\right)^{2}}{\|\bar{\psi}\|^{2}}+\frac{\left(1, \delta q^{\perp}\right)^{2}}{\|1\|^{2}} \geq \theta\left\|\delta q^{\perp}\right\|^{2}
$$


holds for a positive constant $\theta$ depending on the angle between $\bar{\psi}$ and 1 in $L^{2}(\mathcal{X})$.

We now substitute this decomposition into (40) and analyze the resulting terms:

$$
\begin{gathered}
\delta^{2} L_{\sigma, \tau}^{E, \Gamma}(\bar{q})=D_{2}\left(\delta q^{\|}, \delta q^{\|}\right)+2 D_{2}\left(\delta q^{\|}, \delta q^{\perp}\right)+D_{2}\left(\delta q^{\perp}, \delta q^{\perp}\right) \\
+\sigma\left(\bar{\psi}, \delta q^{\perp}\right)^{2}+\tau\left(1, \delta q^{\perp}\right)^{2}
\end{gathered}
$$

The nondegeneracy hypothesis ensures that

$$
D_{2}\left(\delta q^{\|}, \delta q^{\|}\right) \geq \mu\left\|\delta q^{\|}\right\|^{2}
$$

On the other hand, the upper bound (38) gives

$$
\left|D_{2}\left(\delta q^{\perp}, \delta q^{\perp}\right)\right| \leq \nu\left\|\delta q^{\perp}\right\|^{2} .
$$

In a similar fashion the cross term is estimated by means of the Cauchy inequality, giving

$$
2\left|D_{2}\left(\delta q^{\|}, \delta q^{\perp}\right)\right| \leq \nu \epsilon\left\|\delta q^{\|}\right\|^{2}+\frac{\nu}{\epsilon}\left\|\delta q^{\perp}\right\|^{2}
$$

for any $\epsilon>0$. When we use (43), (44), and (45) to estimate the various terms in (42), we obtain the following lower bound:

$$
\begin{gathered}
\delta^{2} L_{\sigma, \tau}^{E, \Gamma}(\bar{q}) \geq \mu\left\|\delta q^{\|}\right\|^{2}-\nu \epsilon\left\|\delta q^{\|}\right\|^{2}-\frac{\nu}{\epsilon}\left\|\delta q^{\perp}\right\|^{2}-\nu\left\|\delta q^{\perp}\right\|^{2} \\
+\sigma\left(\bar{\psi}, \delta q^{\perp}\right)^{2}+\tau\left(1, \delta q^{\perp}\right)^{2}
\end{gathered}
$$

We therefore choose $\epsilon=\mu / 2 \nu$ to make the terms in $\delta q^{\|}$definite. Then, in order to make the terms in $\delta q^{\perp}$ definite, we seek $\sigma$ and $\tau$ so that

$$
\sigma\left(\bar{\psi}, \delta q^{\perp}\right)^{2}+\tau\left(1, \delta q^{\perp}\right)^{2} \geq\left[\frac{\mu}{2}+\frac{\nu}{\epsilon}+\nu\right]\left\|\delta q^{\perp}\right\|^{2}
$$

It suffices to set these penalization parameters so that $\sigma \theta\|\bar{\psi}\|^{2}$ and $\tau \theta\|1\|^{2}$ equal the common value $\mu / 2+\nu / \epsilon+\nu$. With this choice, we obtain the desired lower bound:

$$
\delta^{2} L_{\sigma, \tau}^{E, \Gamma}(\bar{q}) \geq \frac{\mu}{2}\left\|\delta q^{\|}\right\|^{2}+\frac{\mu}{2}\left\|\delta q^{\perp}\right\|^{2}=\frac{\mu}{2}\|\delta q\|^{2} .
$$

Thus, $\delta^{2} L_{\sigma, \tau}^{E, \Gamma}(\bar{q})$ is strictly positive definite, and hence we conclude that for all $q$ in a sufficiently small neigborhood of $\bar{q}$,

$$
\tilde{\mu}\|q-\bar{q}\|^{2} \leq L_{\sigma, \tau}^{E, \Gamma}(q) \leq \tilde{\nu}\|q-\bar{q}\|^{2}
$$

for some $0<\tilde{\mu}<\tilde{\nu}<\infty$. The usual Lyapunov stability argument therefore ensues, since $L_{\sigma, \tau}^{E, \Gamma}$ is a conserved quantity for the dynamics. Thus, the proof of the theorem is complete. 
As in the canonical model, we note that this Lyapunov stability argument remains valid when the objective functional $I$ is treated as a fragile invariant, since constraint functionals $H$ and $C$ are rugged invariants.

We conclude this discussion of stability with some remarks about the role of the penalization in the Lyapunov functional $L_{\sigma, \tau}^{E, \Gamma}$ and its relation to the microcanonical entropy $S(E, \Gamma)$. For the sake of definiteness, let us suppose that $S(E, \Gamma)$ is smooth $\left(C^{2}\right)$ on its domain $\mathcal{A}$, and let us consider a constraint pair $(E, \Gamma)$ that does not belong to $\mathcal{C}$, the concavity set. Then, according to the results in Section 4, the microcanonical equilibrium macrostate $\bar{q}$ for $(E, \Gamma)$ does not belong to any canonical equilibrium set, and the tangent plane to $S$ at $(E, \Gamma)$ is not a supporting plane, meaning that (34) is violated for some $\left(E^{\prime}, \Gamma^{\prime}\right)$. Nevertheless, it is possible to choose constants $\sigma$ and $\tau$ so that they define a supporting paraboloid to $S$ at $(E, \Gamma)$, in the sense that

$$
S\left(E^{\prime}, \Gamma^{\prime}\right) \leq S(E, \Gamma)+\beta\left(E^{\prime}-E\right)+\gamma\left(\Gamma^{\prime}-\Gamma\right)+\frac{\sigma}{2}\left(E^{\prime}-E\right)^{2}+\frac{\tau}{2}\left(\Gamma^{\prime}-\Gamma\right)^{2}
$$

for all $\left(E^{\prime}, \Gamma^{\prime}\right)$ in $\mathcal{A}$, with equality only when $\left(E^{\prime}, \Gamma^{\prime}\right)=(E, \Gamma)$. It follows that $L_{\sigma, \tau}^{E, \Gamma}(q)>$ $L_{\sigma, \tau}^{E, \Gamma}(\bar{q})=0$ for all $q \neq \bar{q}$, by an argument analogous to that used in the proof of Theorem 4 . Thus, we see that the minimal choice of the penalization constants $\sigma$ and $\tau$ is determined by the condition that, at least locally, the corresponding paraboloid lies above the function $S$ and contacts it only at $(E, \Gamma)$.

\section{Numerical example}

6.1 Barotropic flow over topography in a zonal channel. For the purposes of illustrating the general results obtained in Sections 4 and 5, we now present a family of computed solutions to the microcanonical variational principle for a particular choice of domain, topography and prior distribution. We especially focus on the shape of the $S(E, \Gamma)$ surface, since it determines whether the corresponding canonical model is equivalent and whether the Arnold stability criteria apply to the equilibrium states. In view of our results in Section 4 showing that all canonical equilibria are included among the microcanonical equilibria, there is no need to implement a solver for the corresponding canonical variational principle.

We take the domain to be a unit square $\mathcal{X}=\left\{-0.5<x_{1}<0.5,-0.5<x_{2}<0.5\right\}$, which represents a normalized zonal channel. For the topography term $b$ in the potential vorticity expression (21), we choose a simple sinusoid, $b=b\left(x_{2}\right)=B_{2} \sin \left(2 \pi x_{2}\right)$. This topography is zonal, being independent of $x_{1}$, and consists of the second harmonic with respect to $x_{2}$.

Such a zonal domain and topography can be viewed as an idealized and simplified model of a zone-belt domain in a Jovian atmosphere [26, 16, 34]. In the 1-1/2-layer model, $b$ is the effective topography that results from an underlying steady mean flow in a deep lower layer. 
The domain is composed of a zone, where $b$ is positive, and a belt, where $b$ is negative. If the amplitude $B_{2}$ of the topography is large enough, one expects that the mean flow in the shallow upper layer will a shear flow $v=\left(v_{1}\left(x_{2}\right), 0\right)$, and that it will tend to be anticyclonic (negative vorticity) in the zone and cyclonic (positive vorticity) in the belt. In our computations of most probable flows we set $B_{2}=1$, and we find that they are zonal shear flows with the expected topography-induced tendencies.

We illustrate the effect of a large or a small radius of deformation by choosing the representative values $r=\infty$ or $r=0.2$. The small deformation radius regime is the one relevant to a Jovian atmosphere [34].

With these choices of the geometrical parameters, the formulation of the model problem is complete once we specify a prior distribution $\rho$, which determines the probabilistic structure of the small-scale potential vorticity $Q$. We select a family of gamma distributions $\rho_{\epsilon}(d y)$ with mean, variance and skewness normalized as follows:

$$
\int y \rho_{\epsilon}(d y)=0, \quad \int y^{2} \rho_{\epsilon}(d y)=1, \quad \int y^{3} \rho_{\epsilon}(d y)=2 \epsilon
$$

the variable $y$ runs through the range of $Q$. For small $\epsilon$, these distributions are close to the standard normal distribution, which they approach in the limit as $\epsilon$ goes to zero. For positive $\epsilon$, they are supported on the interval $-\epsilon^{-1} \leq y<+\infty$, and they have an exponential tail in the positive $y$-direction. They are defined explicitly by the probability density

$$
\rho_{\epsilon}(d y)=\frac{1}{\epsilon \Gamma\left(\epsilon^{-2}\right)} \exp \left(\epsilon^{-2}[\log (1+\epsilon y)-(1+\epsilon y)]\right) d y .
$$

The family of prior distributions $\rho_{\epsilon}(d y)$ have the virtue that their cumulant generating functions $f_{\epsilon}(\eta)$, which are defined by $(13)$, can be calculated explicitly; namely,

$$
\begin{aligned}
f_{\epsilon}(\eta) & =-\epsilon^{-1} \eta-\epsilon^{-2} \log (1-\epsilon \eta) \\
& =\eta^{2} / 2+\epsilon \eta^{3} / 3+O\left(\epsilon^{2}\right) .
\end{aligned}
$$

The associated information functional $I_{\epsilon}$, defined in (15), is then determined by the conjugate function $i_{\epsilon}(y)$ to $f_{\epsilon}(\eta)$; namely,

$$
i_{\epsilon}(y)=\epsilon^{-1} y-\epsilon^{-2} \log (1+\epsilon y) .
$$

The relevant properties of the convex function $i_{\epsilon}(y)$ are easily seen from its second derivative, $i_{\epsilon}^{\prime \prime}(y)=(1+\epsilon y)^{-2}$.

The mean-field equation (25) corresponding to this choice of prior distribution is

$$
\begin{aligned}
\bar{q}=-\Delta \bar{\psi}+r^{-2} \bar{\psi}+B_{2} \sin 2 \pi x_{2} & =\epsilon^{-1}\left([1-\epsilon(-\beta \bar{\psi}-\gamma)]^{-1}-1\right) \\
& =(-\beta \bar{\psi}-\gamma)+\epsilon(-\beta \bar{\psi}-\gamma)^{2}+O\left(\epsilon^{2}\right) .
\end{aligned}
$$


¿From the above expansion it is evident that $\epsilon$ determines the magnitude of the principal nonlinear term in this equation. When $\epsilon=0$, the models resemble the so-called energyenstrophy theory, in which the statistical equilibrium distributions are Gaussian and the mean-field equations are linear [30, 46, 9, 32]. For the sake of definiteness, we fix $\epsilon=0.1$ in the computations to follow. It is worth noting that $\epsilon$ links the skewness of the prior distribution to the nonlinearity of the mean-field equation.

While many reasonable choices of prior distribution suffice for the purposes of the present example, the relation between the potential vorticity and streamfunction in (48) is distinguished by the fact that it agrees with the form of the relation inferred by an analysis of observed zonal winds on Jupiter [16]. We note however that this physically interesting prior distribution violates the growth condition (11) assumed for simplicity in our discussion of the general theory. Nevertheless, all of the key results described in the preceding sections remain valid for this prior distribution, although their proofs are somewhat more involved. In particular, the basic large deviation principle given in Theorem 1 continues to hold; by virtue of the Gartner-Ellis Theorem [12], it is sufficient that $f_{\epsilon}(\eta)$ is finite and smooth on the interval $-\infty<\eta<\epsilon^{-1}$. We omit the analysis that justifies this extension of the theory already developed.

6.2 Computed results. To solve the variational principle for the microcanonical model derived in Theorem 3, we implement the iterative algorithm developed in [50] and extended in [15]. Specifically, for given admissible values $E$ and $\Gamma$ of the energy and circulation constraints, respectively, we compute the equilibrium macrostate $\bar{q}$ that solves

$$
\text { minimize } I_{\epsilon}(q) \quad \text { subject to } H(q)=E, C(q)=\Gamma \text {. }
$$

¿From an initial guess $q^{0}$ having the given constaint values $(E, \Gamma)$, this algorithm defines a sequence $q^{k}$ of approximations that converges to a solution $\bar{q}$ as $k \rightarrow \infty$. At each iteration, a variational subproblem defined by linearizing the energy constraint is solved; its solution, $q^{k}$, then satisfies $H\left(q^{k}\right) \geq E, C\left(q^{k}\right)=\Gamma$, and $I\left(q^{k}\right) \leq I\left(q^{k-1}\right)$. These properties of the iteration step guarantee that the algorithm is globally convergent [50]. In the limit as $k \rightarrow \infty$, the equality constraint on energy is retrieved, and the iterative multipliers, $\beta^{k}$ and $\gamma^{k}$, which are determined along with $q^{k}$, converge to the multipliers $\beta$ and $\gamma$ associated with $\bar{q}$. Experience with this algorithm in a wide range of statistical equilibrium problems has shown it to be an efficient and robust method.

We now turn to a description of the computed results for this specific microcanonical equilibrium problem.

We compute the equilibrium states $\bar{q}=\bar{q}\left(x_{2} ; E, \Gamma\right)$ over the range of constraint values, $0<E \leq 0.1,-2 \leq \Gamma \leq 2$, for both (a) $r=\infty$ and (b) $r=0.2$. In each case, we tabulate the microcanonical entropy $S(E, \Gamma)=-I(\bar{q})$. In Figure 1 , we exhibit the admissible set $\mathcal{A}$ and the concavity set $\mathcal{C}$ for these two values of $r$. We recall from Section 4 that $\mathcal{A}$ is the set of all pairs $(E, \Gamma)$ for which there exists some macrostate $q$ realizing those constraint values 
$(E, \Gamma)$, and that $\mathcal{C}$ is the subset of all pairs $(E, \Gamma)$ at which $S$ has a supporting plane. In Figure $1, \mathcal{C}$ is indicated by "equivalence" and $\mathcal{A} \backslash \mathcal{C}$ by "nonequivalence." The remarkable result contained in Figure 1 is that, for both $r=\infty$ and $r=0.2$, the concavity set $\mathcal{C}$ is a relatively small subset of the admissible set $\mathcal{A}$. In fact, for any fixed circulation $\Gamma$, the pair $(E, \Gamma)$ lies in $\mathcal{C}$ only for a limited range of energies near the smallest admissible energy. For all the energies outside this range, the tangent plane to $S$ at $(E, \Gamma)$ is not a supporting plane for $S$. Consequently, for this range of larger energies, the equivalence of ensembles breaks down, meaning that the canonical model omits all these microcanonical equilibrium states.

Another graphical depiction of the nonconcavity present in $S(E, \Gamma)$ is given in Figures 2 and 3. In Figure 2 the section of $S$ versus $E$ at the fixed value $\Gamma=0$ is plotted. This entropy-energy curve shows that the inverse temperature $\beta=\partial S / \partial E$ is positive only for a small range of low energies below $E=0.01$. Throughout the negative temperature range, the entropy function is slightly concave with respect to $E$, becoming asymptotically linear for high energy values. By contrast, the section of $S$ versus $\Gamma$ at $E=0.05$ plotted in Figure 3 shows that the entropy-circulation curve is strongly nonconcave for a wide range of circulation values around zero. This result suggests that in this particular problem the nonequivalence of ensembles is largely a consequence of the circulation constraint.

Figures 1, 2 and 3 also indicate the dependence of the solutions on the radius of deformation $r$. The nonequivalence set $\mathcal{A} \backslash \mathcal{C}$ broadens noticably as $r$ is decreased from $r=\infty$ to $r=0.2$. Also, the asymmetry in the entropy-circulation curve, which is a consequence of the skewness $2 \epsilon$ of the prior distribution, increases with decreasing $r$. These two results suggest that the effect of the nonlinearity, as measured by $\epsilon$, is strengthened by a small deformation radius. From this behavior we conclude that the breakdown of the equivalence of ensembles is exacerbated by a weak vertical stratification, which results in a small $r$. This conclusion is especially interesting in the application to the Jovian atmosphere, where large-scale mean flows such as the permanent zonal winds typically span several radii of deformation [34].

Finally, in Figure 4 we display the mean velocity fields associated with some representative microcanonical equilibrium states. Specifically, we fix $r=0.2$ and $E=0.05$, and we choose three representative values of the circulation: (a) $\Gamma=-0.5$; (b) $\Gamma=1.4$; (c) $\Gamma=2.0$. Flow (a) lies within the nonequivalance set, near the local minimum of $S$ with respect to $\Gamma$; flow (b) lies near the equivalence-nonequivalence boundary, which itself is near the global maximum of $S$ with respect to $\Gamma$; flow (c) lies in the equivalence set. We draw particular attention to flow (a), which closely resembles the mean zonal winds observed in a zone-belt domain of the Jovian atmosphere. Indeed, this shear flow consists of a strong westward jet that resides between two strong eastward jets. Furthermore, even though the prior distribution has a positive (cyclonic) skewness, this intense triple-jet flow has a negative (anticyclonic) circulation. Interestingly, most of the coherent structures observed on Jupiter and the other giant planets are anticyclonic. These general properties on flow (a), which is representative of the most probable flows in the nonequivalence set, are not 
shared by flows (b) and (c). Instead, each of these flows consists of one broad westward jet and one narrow eastward jet, and the total circulation of each of them is positive (cyclonic). These weaker shear flow structures are typical of the equivalence set.

Perhaps our most significant result is that the most probable flows corresponding to a constraint pairs (E.Г) in the nonequivalence set are nonlinearly stable, even though they typically fail to satisfy the well-known stability conditions. The computed flows discussed above illustrate this general result quite vividly. The most probable flows (a) and (b) displayed in Figure 4 have negative $\beta$ and fail the often-quoted sufficient condition

$$
0<\frac{d \bar{q}}{d \bar{\psi}}<\lambda_{1}
$$

for the second Arnold stability theorem. Indeed, our computations show that for the triplejet flow (a), $d \bar{q} / d \bar{\psi}$ ranges from 27 and 78 , while for the qualitatively different flow (b), $d \bar{q} / d \bar{\psi}$ ranges from 26 to 42 . Since $\lambda_{1}=\pi^{2}+r^{-2} \approx 35$, we conclude that flow (a), which lies within the nonequivalence set, is far from satisfying (49), while flow (b), which lies near the equivalence-nonequivalence boundary, comes closer to fulfilling (49).

By contrast, flow (c) in Figure 4, which is a positive temperature macrostate lying in the equivalence set, satisfies the Rayleigh condition

$$
\frac{d \bar{q}}{d \bar{\psi}}<0
$$

which implies the first Arnold stability theorem. In fact, for flow (c), $d \bar{q} / d \bar{\psi}$ is approximately equal to the constant -5 over the domain.

Let us comment further on this gap in the classical stability criteria. First, any microcanonical equilibrium $\bar{q}$, which corresponds to a constraint pair $(E, \Gamma)$ belonging to the equivalence set $\mathcal{C}$, is a global minimizer of the associated information functional $I_{\beta, \gamma}$. Thus, in principle, the classical Arnold stability criterion applies, assuming only that the minimizer is nondegenerate. Nevertheless, it is possible that the explicit sufficient condition (49) may be violated, even though $I_{\beta, \gamma}$ is a Lyapunov functional for $\bar{q}$. Second, in the case of an equilibrium $\bar{q}$ which lies slightly outside the equivalence set, it is possible that the $I_{\beta, \gamma}$ is a Lyapunov functional, if the microcanonical entropy $S(E, \Gamma)$ is locally concave at $\bar{q}$; then, the tangent plane corresponding to $(\beta, \gamma)$ is locally a supporting plane for $S$, even though it does not support $S$ globally. Typically, the sufficient condition (49) is too crude in such a delicate case. Third, for an equilibrium $\bar{q}$ which lies far outside the equivalence set, $I_{\beta, \gamma}$ is not definite at $\bar{q}$, and the classical Lyapunov argument based on this functional fails. Of course, in this nonequivalent case the sufficient condition (49) is violated.

The above analysis of the various cases possible in the classical stability criteria notwithstanding, Theorem 7 guarantees that the microcanonical equilibrium states corresponding to all admissible pairs $(E, \Gamma)$ define nonlinearly stable flows, provided only that a technical nondegeneracy condition is fulfilled. Given this refined stability result, which makes use of 
the penalized Lyapunov functional $L_{\sigma, \tau}^{E, \Gamma}$, it is not necessary to impose a restrictive condition such as (49) to obtain the stability of most probable flows. Conversely, it is incorrect to assume that a steady flow that strongly violates the well-known Arnold conditions is unstable. In essence, these conditions are derived by utilizing a linear combination of two independent conserved quantities (the energy and a certain enstrophy), while the conservation of each of these quantities separately constraints the evolution of perturbations and leads to more refined stability conditions.

\section{Acknowledgements}

The authors thank Michael Kiessling and Andrew Majda for helpful conversations that stimulated aspects of this work.

\section{References}

[1] V. Arnold. Mathematical Methods in Classical Mechanics. Springer-Verlag, New York, 1982.

[2] R. Balescu, Equilibrium and Nonequilibrium Statistical Mechanics. Wiley, New York, 1975.

[3] R. Balian. From Microphysics to Macrophysics: Methods and Applications of Statistical Physics, vol. 1. Springer-Verlag, New York, 1982.

[4] D. P. Bertsekas. Constrained optimization and Lagrange multiplier methods, Academic Press, New York, 1982.

[5] C. Boucher, R. S. Ellis, and B. Turkington. "Derivation of maximum entropy principles in two-dimensional turbulence via large deviations." J. Stat. Phys. 98:1235-1278, 2000.

[6] H. Brands, J. Stulemeyer, and R. A. Pasmanter, "A mean field prediction of the asymptotic state of decaying 2D turbulence." Phys. Fluids A 9:2815, 1997.

[7] F. P. Bretherton and D. B. Haidvogel. "Two-dimensional turbulence over topography." J. Fluid Mech. 78:129-154, 1976.

[8] E. Caglioti, P. L. Lions, C. Marchioro, and M. Pulvirenti. "A special class of stationary flows for two-dimensional Euler equations: a statistical mechanics description." Commun. Math. Phys. 143:501-525, 1992.

[9] G. Carnavale and J. Frederiksen, "Nonlinear stability and statistical mechanics of flow over topography." J. Fluid Mech. 175:157-181, 1987. 
[10] A. J. Chorin. Vorticity and Turbulence. New York: Springer-Verlag, 1994.

[11] A. J. Chorin. "Partition functions and equilibrium measures in two-dimensional and quasi-three-dimensional turbulence." Phys. Fluids 8:2656-2660, 1996.

[12] A. Dembo and O. Zeitouni. Large Deviations Techniques and their Applications. Boston: Jones \& Bartlett, 1993.

[13] M. DiBattista and A. Majda. "An equilibrium statistical model for the spreading phase of open-ocean convection." Proc. Nat. Acad. Sci. 96:6009-6013, 1999.

[14] M. DiBattista and A. Majda. "Meta-stability of equilibrium statistical structures for prototype geophysical flows with damping and driving." to appear in Physica D.

[15] M. DiBattista, A. Majda and B. Turkington. "Prototype geophysical vortex structures via large-scale statistical theory." Geophys. Astrophys. Fluid Dyn., 89:235-283, 1998.

[16] T. E. Dowling. "A relationship between potential vorticity and zonal winds on Jupiter." J. Atmos. Sci. 50:14-22, 1993.

[17] T. E. Dowling and A. P. Ingersoll. "Jupiter's Red Spot as a shallow water system." J. Atmos. Sci. 46:3256-3278, 1989.

[18] R. S. Ellis. Entropy, Large Deviations, and Statistical Mechanics. Springer-Verlag, 1985.

[19] R. S. Ellis. "An overview of the theory of large deviations and applications to statistical mechanics." Scand. Actuarial J. No. 1:97-142, 1995.

[20] R. S. Ellis, K. Haven, and B. Turkington. "Large deviation principles and complete equivalence and nonequivalence results for pure and mixed ensembles." Preprint, 2000.

[21] R. S. Ellis, K. Haven, and B. Turkington. "The large deviation principle for coarsegrained processes." Preprint, 2000.

[22] G. L. Eyink and H. Spohn. "Negative-temperature states and large-scale, long-lived vortices in two-dimensional turbulence." J. Statist. Phys. 70:833-886, 1993.

[23] M. J. Grote and A. Majda. "Crude closure dynamics through large scale statistical theories." Phys. Fluids. 9:3431-3442, 1997.

[24] G. Holloway. "Eddies, waves, circulation and mixing: statistical geofluid mechanics." Annual Rev. Fluid Mech. 18:91-147, 1986.

[25] D. Holm, J. Marsden, T. Ratiu, and A. Weinstein. "Nonlinear stability of fluid and plasma equilibria." Rev. Mod. Phys. 123:1-116, 1985. 
[26] A. P. Ingersoll and P. G. Cuong. "Numerical model of long-lived Jovian vortices." J. Atmos. Sci. 38:2067-2076, 1981.

[27] A. D. Ioffe and V. M. Tihomirov. Theory of Extremal Problems. New York: Elsevier North-Holland, 1979.

[28] G. Joyce and D. C. Montgomery. "Negative temperature states for the two-dimensional guiding center plasma." J. Plasma Phys. 10:107-121, 1973.

[29] M. Kiessling. "Statistical mechanics of classical particles with logarithmic interactions." Commun. Pure Appl. Math. 46:27-56, 1993.

[30] R. Kraichnan. "Statistical dynamics of two-dimensional flow." J. Fluid Mech. 67:155$175,1975$.

[31] J. C. McWilliams. "The emergence of isolated coherent vortices in turbulent flow." J. Fluid Mech. 146:21-43, 1984.

[32] A. Majda and M. Holen. "Dissipation, topography, and statistical theories of largescale coherent structures." Commun. Pure Appl. Math. 50:1183-1234, 1997.

[33] C. Marchioro and M. Pulvirenti. Mathematical Theory of Incompressible Nonviscous Fluids. Appl. Math. Sci., volume 96. New York: Springer-Verlag, 1994.

[34] P. S. Marcus. "Jupiter's Great Red Spot and other vortices." Annual Rev. Astrophys. 31:523-273, 1993.

[35] J. Michel and R. Robert. "Large deviations for Young measures and statistical mechanics of infinite dimensional dynamical systems with conservation law." Commun. Math. Phys. 159:195-215, 1994.

[36] J. Miller. "Statistical mechanics of Euler equations in two dimensions." Phys. Rev. Lett. 65:2137-2140, 1990.

[37] J. Miller, P. Weichman, and M. C. Cross. "Statistical mechanics, Euler's equations, and Jupiter's red spot." Phys. Rev. A 45:2328-2359, 1992.

[38] M. Minoux, Mathematical Programing: Theory and Algorithms, Wiley-Interscience, John-Wiley and Sons, Chichester, 1986.

[39] D. Montgomery, W. T. Matthaeus, D. Martinez, and S. Oughton. "Relaxation in two dimensions and the Sinh-Poisson equation." Phys. Fluids A 4:3-6, 1992.

[40] L. Onsager. "Statistical hydrodynamics." Suppl. Nuovo Cim. 6:279-287, 1949.

[41] J. Pedlosky. Geophysical Fluid Dynamics. Springer, 1979. 
[42] L. M. Polvani, J. C. McWilliams, M. A. Spall and R. Ford, "The coherent structures of shallow-water turbulence: Deformation-radius effects, cyclone/anticyclone asymmetry and gravity-wave generation," Chaos 4(2): 177-186, 1994.

[43] P. Rhines. "Geostrophic turbulence." Annual Rev. Fluid Mech. 11:404-441, 1979.

[44] R. Robert. "A maximum-entropy principle for two-dimensional perfect fluid dynamics." J. Statist. Phys. 65:531-553, 1991.

[45] R. Robert and J. Sommeria. "Statistical equilibrium states for two-dimensional flows." J. Fluid Mech. 229:291-310, 1991.

[46] R. Salmon, G. Holloway and M. C. Henderschott. "The equilibrium statistical mechanics of simple quasi-geostrophic models." J. Fluid Mech. 75:691-703, 1976.

[47] P. Santangelo, R. Benzi, and B. Legras. "The generation of vortices in high-resolution, two-dimensional decaying turbulence and the influence of initial conditions on the breaking of self-similarity." Phys. Fluids A 1:1027-1034, 1989.

[48] E. Segre and S. Kida. "Late states of incompressible 2D decaying vorticity fields." Fluid Dyn. Res. 23:89-112 (1998).

[49] B. Turkington. "Statistical equilibrium measures and coherent states in twodimensional turbulence." Commun. Pure Appl. Math. 52:781-809, 1999.

[50] B. Turkington and N. Whitaker, "Statistical equilibrium computations of coherent structures in turbulent shear layers." SIAM J. Sci. Comput. 17:1414-1433, 1996.

[51] E. Zeidler. Nonlinear Functional Analysis and its Applications III: Variational Methods and Optimization. New York: Springer-Verlag, 1985. 


\section{Figure captions}

Fig. 1. Admissible set $\mathcal{A}$ and concavity set $\mathcal{C}$ for the microcanonical variational principle for a range of constraint values on energy $(0.01 \leq E \leq 0.1)$ and circulation $(-2 \leq \Gamma \leq 2)$. The computed boundary of the admissible set is the dashed curve; the computed boundary of the concavity, or equivalence, set is the solid curve. For each admissible constraint pair $(E, \Gamma)$ in a grid over this range with $\Delta E=0.0025$ and $\Delta \Gamma=0.025$, the corresponding equilibrium macrostate $\bar{q}$, multipliers $\beta$ and $\gamma$, and microcanonical entropy $S$ are computed. A pair $(E, \Gamma)$ is accepted for the concavity set if the tangent plane at $\bar{q}$ with slopes $\beta$ and $\gamma$ lies above the function $S$ throughout the admissible set. This computation is displayed for two different choices of deformation radius: (a) $r=\infty$ and (b) $r=0.2$.

Fig. 2. The section $S(E, 0)$ of the microcanonical entropy for the same variational problem as in Figure 1. The solid curve is for (a) $r=\infty$, and the dashed curve is for (b) $r=0.2$.

Fig. 3. The section $S(0.05, \Gamma)$ of the microcanonical entropy for the same variational problem as in Figure 1. The solid curve is for (a) $r=\infty$, and the dashed curve is for (b) $r=0.2$.

Fig. 4. Mean velocity fields of the zonal shear flows determined by the most probable macrostates for the microcanonical model with $r=0.2$ and $E=0.05$. Flows corresponding to three different circulations are displayed: (a) $\Gamma=-0.5$, which lies within the nonequivalence set; (b) $\Gamma=1.4$, which lies near the equivalence-nonequivalence boundary; (c) $\Gamma=2.0$, which lies in the equivalence set. 
(a) $r=\infty$

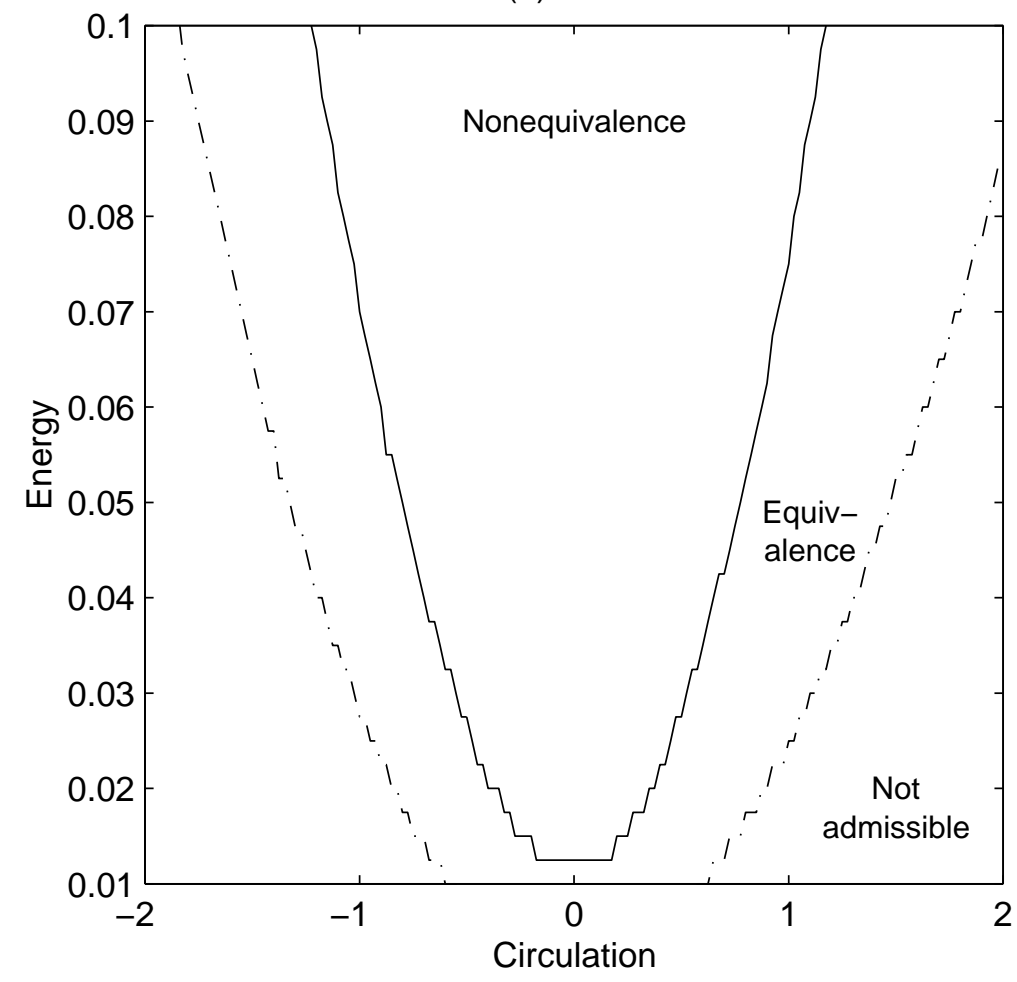

(b) $r=0.2$

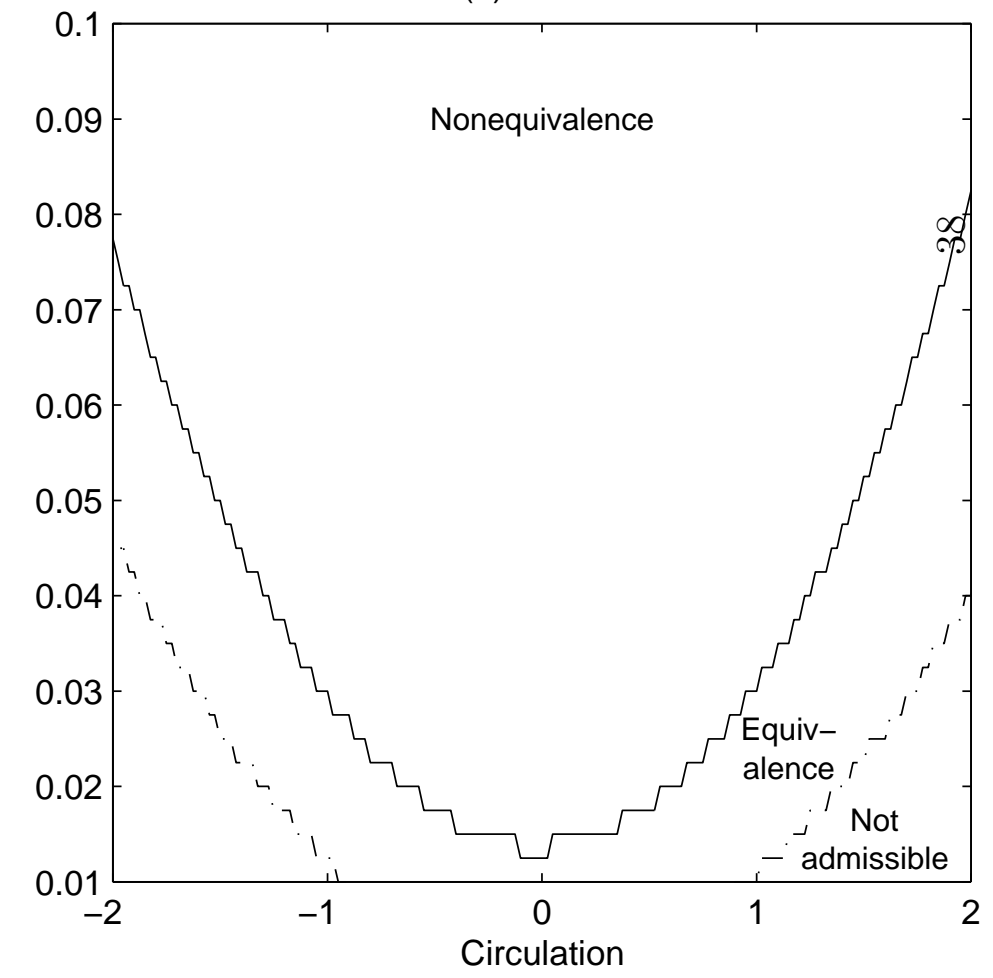




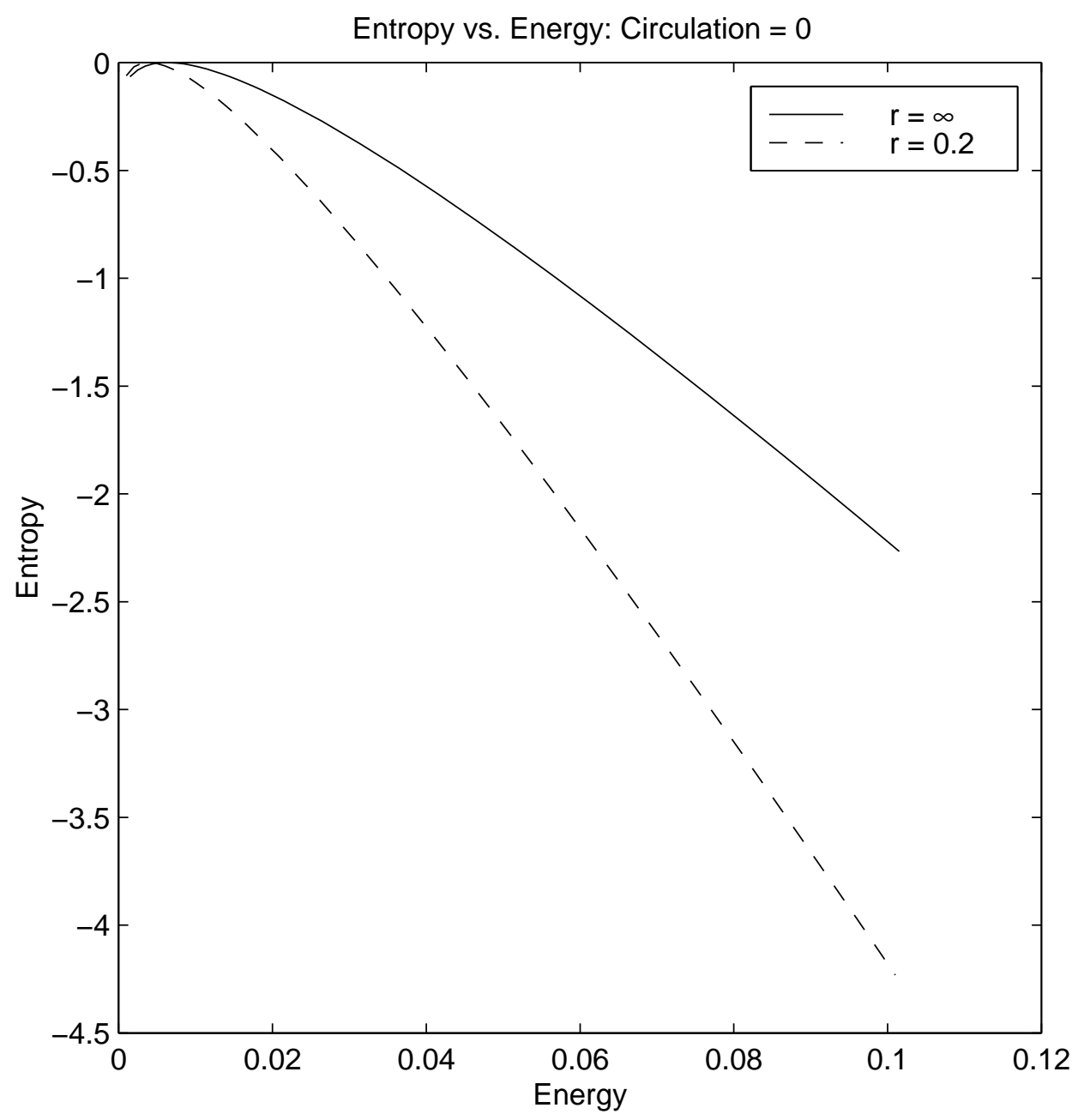




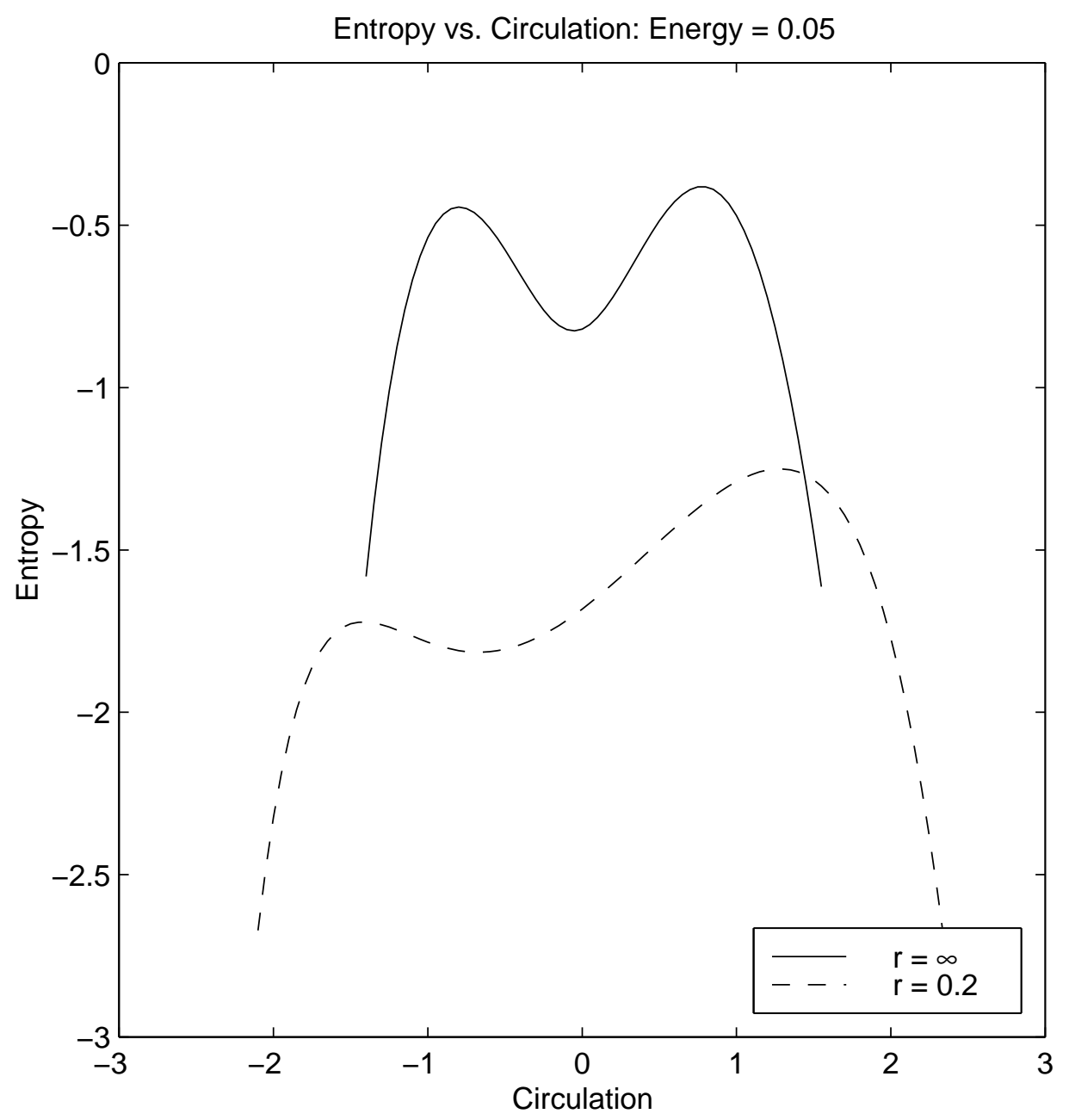


(a) $E=0.05, \Gamma=-0.5$

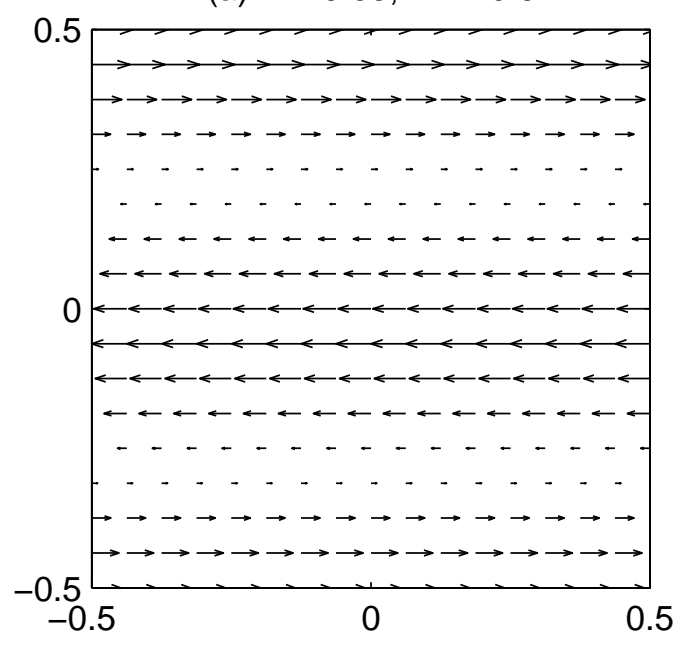

(b) $E=0.05, \Gamma=1.4$

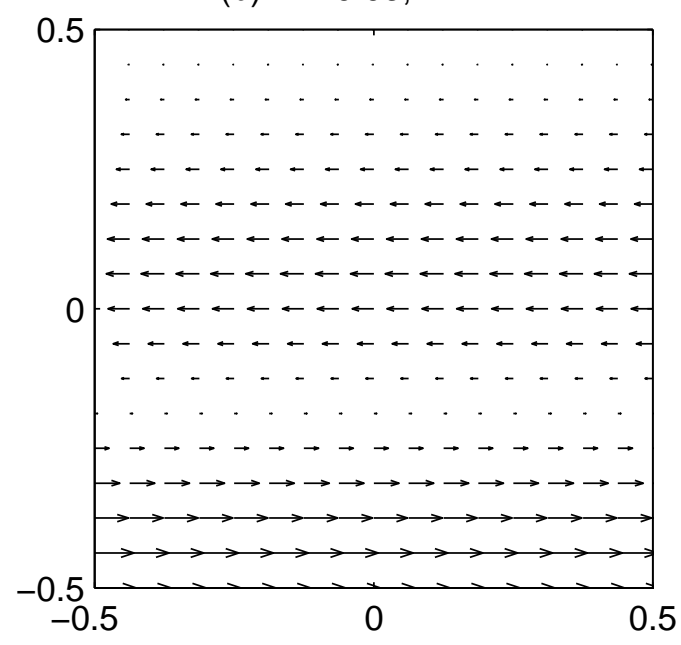

(c) $E=0.05, \Gamma=2.0$

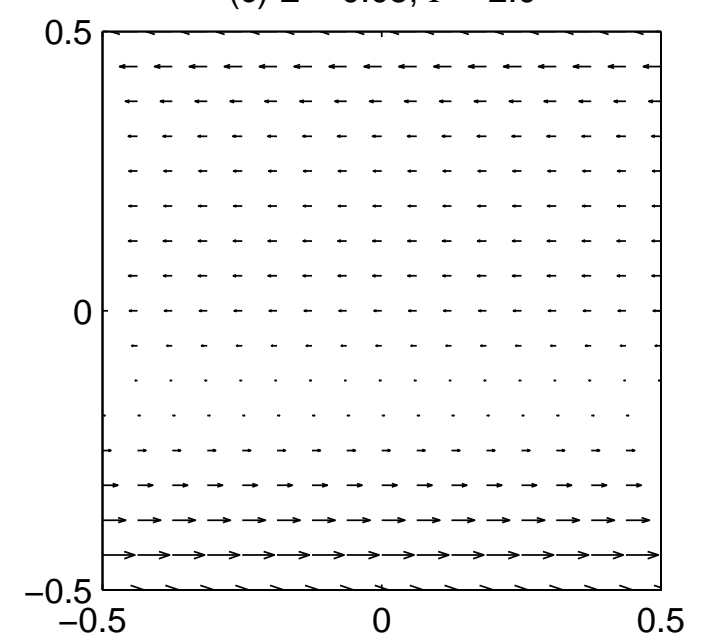

